\title{
An alternative existence proof of the geometry of Ivanov-Shpectorov for O'Nan's sporadic group
}

\author{
Francis Buekenhout Thomas Connor \\ In honor of J. A. Thas's 70th birthday
}

\begin{abstract}
We provide an existence proof of the Ivanov-Shpectorov rank 5 diagram geometry together with its boolean lattice of parabolic subgroups and establish the structure of hyperlines.
\end{abstract}

Keywords : incidence geometry, diagram geometry, Buekenhout diagrams, O'Nan's sporadic group

MSC 2010: 51E24, 20D08, 20B99

\section{Introduction}

We start essentially but not exclusively from:

- Leemans [26] giving the complete partially ordered set $\Lambda_{\mathrm{O}^{\prime} \mathrm{N}}$ of conjugacy classes of subgroups of the O'Nan group $\mathrm{O}^{\prime} \mathrm{N}$. This includes 581 classes and provides a structure name common for all subgroups in a given class;

- the Ivanov-Shpectorov [24] rank 5 diagram geometry for the group $\mathrm{O}^{\prime} \mathrm{N}$, especially its diagram $\Delta$ as in Figure 1;

- the rank 3 diagram geometry $\Gamma_{\mathrm{Co}}$ for $\mathrm{O}^{\prime} \mathrm{N}$ due to Connor [15];

- detailed data on the diagram geometries for the groups $\mathrm{M}_{11}$ and $\mathrm{J}_{1}[13$, $6,27]$. 


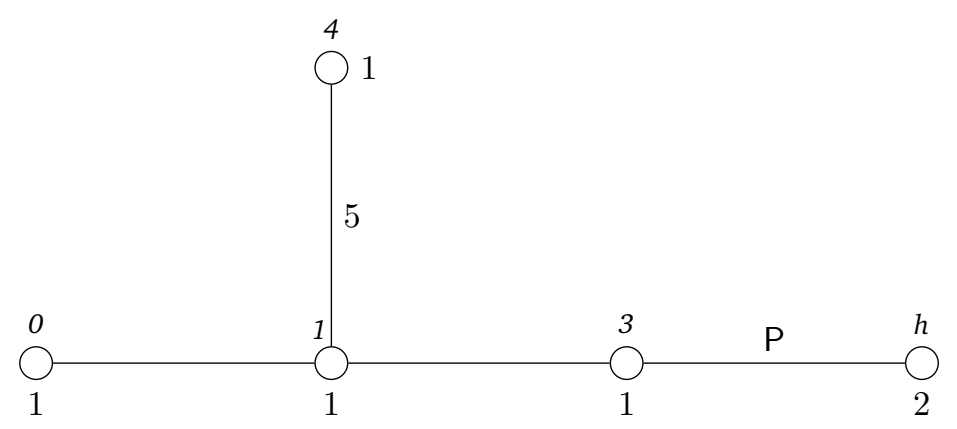

Figure 1: The diagram $\Delta_{\text {IvSh }}$ of the geometry $\Gamma_{\text {IvSh }}$

Our results are the following:

- we get the Connor geometry $\Gamma_{\mathrm{Co}}$ as a truncation of the Ivanov-Shpectorov geometry $\Gamma_{\text {IvSh }}$ (see Theorem 7.1);

- using the paper of Ivanov and Shpectorov [24], we establish the full structure of the boolean lattice $L_{\mathrm{IvSh}}$ of their geometry as in Figure 17 (See Section 8);

- conversely, within $\Lambda_{\mathrm{O}^{\prime} \mathrm{N}}$ we prove the existence and uniqueness up to fusion in $\operatorname{Aut}\left(\mathrm{O}^{\prime} \mathrm{N}\right)$ of a boolean lattice isomorphic to $L_{\mathrm{IvSh}}$. This step is independent of [24] (see Theorem 8.1);

- we prove that this boolean lattice yields a rank 5 diagram geometry $\Gamma_{\text {IvSh }}$ and so establish the existence of the Ivanov-Shpectorov geometry (see Theorem 9.4);

- using the 0-elements of $\Gamma_{\text {IvSh }}$ as in Figure 1 and calling them points, we show that every $h$-element deserves the name hyperline (see Theorem 10.5).

In 1986 Ivanov and Shpectorov gave the construction of a geometry $\Gamma_{\text {IvSh }}$ of rank 5 on which the O'Nan group acts flag-transitively [24]. It belongs to the diagram given in Figure 1 in which our set of types is $\{0,1,3,4, h\}$. Motivation for this seemingly strange set of types will be given in Section 5 . Their work provided the existence proof of two conjugacy classes of subgroups isomorphic to $\mathrm{M}_{11}$ in $\mathrm{O}^{\prime} \mathrm{N}$. The paper is not easy to read and it does not seem to have given rise to more detailed versions. In several steps we provide a new approach to this geometry which is broadly independent from the original paper. In a recent paper Connor [15] constructed a new coset geometry $\Gamma_{\mathrm{Co}}$ for the O'Nan 
sporadic simple group which is of rank 3 over the diagram of Figure 2. The construction is based on a convenient amalgam of known rank 2 coset geometries for the sporadic groups $\mathrm{J}_{1}$ and $\mathrm{M}_{11}$. His finding was based on the subgroup lattice for $\mathrm{O}^{\prime} \mathrm{N}$ as provided by Leemans [26]. Connor's idea was to extend the boolean lattices of the rank 2 geometries for $\mathrm{J}_{1}$ and $\mathrm{M}_{11}$ in a rank 3 boolean lattice of subgroups of $\mathrm{O}^{\prime} \mathrm{N}$. It turned out that there is a unique solution to this problem up to conjugacy. Applying a theorem due to Aschbacher [1], he got the existence of this new flag-transitive coset geometry and additional properties.

Using the diagram of $\Gamma_{\text {IvSh }}$ alone we show that $\Gamma_{\text {Co }}$ is a truncation of $\Gamma_{\text {IvSh }}$ (Theorem 7.1). It matters to state that $\Gamma_{\text {Co }}$ was constructed and studied without making use of $\Gamma_{\text {IvSh }}$. From this fact it is conceivable to extend the Connor geometry with elements so as to produce the Ivanov-Shpectorov geometry. In order to do so we show that $\Gamma_{\text {Co }}$ and the diagram of Figure 1 determine uniquely $\Gamma_{\text {IvSh }}$ (Theorems 8.1 and 9.4 combined). A major step of this characterization makes use of Leemans's subgroup lattice of $\mathrm{O}^{\prime} \mathrm{N}$ [26]. We use it to show that the diagram of Figure 1 and the boolean lattice of $\Gamma_{\text {Co }}$ determine a unique boolean lattice of rank 5 in $\mathrm{O}^{\prime} \mathrm{N}$. The final step is to use this boolean lattice in order to construct a geometry whose diagram is exactly the one of Figure 1 . We provide all of this in full detail. In this way we produce an alternative existence proof for the Ivanov-Shpectorov geometry. Our main result is stated as follows.

Theorem 1.1. Up to conjugacy in Aut $\left(\mathrm{O}^{\prime} \mathrm{N}\right)$, there exists a unique boolean lattice of rank 5 in the subgroup lattice of $\mathrm{O}^{\prime} \mathrm{N}$ as in Figure 17. This boolean lattice defines a unique firm, residually connected, flag-transitive geometry of rank 5 over the diagram of Figure 1.

The first part of this theorem is Theorem 8.1 and the second part is Theorem 9.4.

The paper is organized as follows. In Section 2 we provide the definitions needed to understand this paper. In Section 4 we give the constructions of two rank 4 geometries that are residues of $\Gamma_{\text {IvSh }}$. In Section 7 we prove that the Connor geometry $\Gamma_{\mathrm{Co}}$ is a truncation of the Ivanov-Shpectorov geometry $\Gamma_{\mathrm{IvSh}}$. In Section 8 we extend uniquely the boolean lattice of $\Gamma_{\mathrm{Co}}$ to a rank 5 boolean lattice in the subgroup lattice of $\mathrm{O}^{\prime} \mathrm{N}$. In Section 9 we show that this extended boolean lattice provides a unique flag-transitive geometry that is $\Gamma_{\text {IvSh }}$. In Section 10 we thoroughly detail structural properties of the $h$-elements of $\Gamma_{\text {IvSh }}$ that we call hyperlines. 


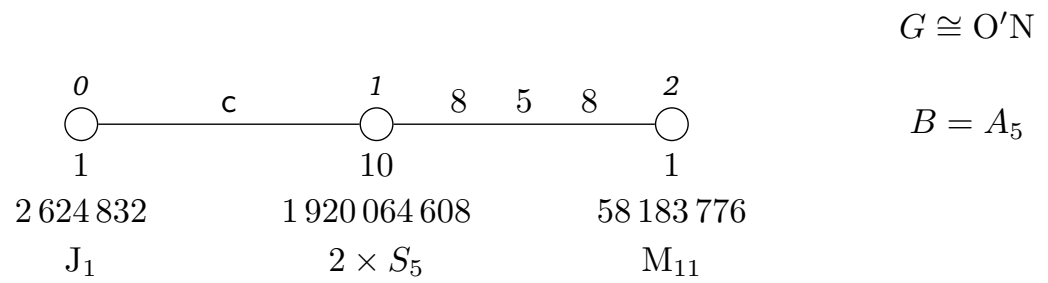

Figure 2: The diagram of the geometry $\Gamma_{\mathrm{Co}}$

\section{Definitions and notations about graphs and inci- dence (resp. coset, diagram) geometries}

In this section we define the notions that are needed to understand our paper. It includes explanations about the meaning of diagrams and their symbols. Our reference for this section is [10].

\subsection{Graphs}

We formalize the notion of a graph and we fix notation as well as terminology.

A graph $\mathcal{G}$ is a pair $(V, E)$ where $V$ is a set whose elements are called vertices and $E$ is a set of pairs of distinct elements of $V$. Elements of $E$ are called edges.

A graph in this sense is commonly called simple, i.e. with no loop nor multiedge. However we only deal with simple graphs and there is no possible ambiguity by omitting the adjective 'simple'.

We call $V$ the vertex-set of $\mathcal{G}$ and $E$ the edge-set of $\mathcal{G}$. The edge-set $E$ of a graph $\mathcal{G}$ defines a symmetric relation on $\sim: V \times V$. We call such a relation an adjacency relation. The data of $V$ and $E$ to define $\mathcal{G}$ is equivalent to the data of $V$ together with the adjacency relation $\sim$. For the sake of simplification, we make the following abuse of terminology: we say that a vertex $v$ of $V$ belongs to $\mathcal{G}$, and we write $v \in \mathcal{G}$ for that.

Given a vertex $v \in \mathcal{G}$, we call the set $\mathrm{N}_{\mathcal{G}}(v):=\{w \in \mathcal{G} \mid v \sim w\}$ the neighborhood of $v$ (where the subscript $\mathcal{G}$ can be omitted if there is no possible confusion). The elements of this set are the neighbors of $v$. We also denote $\mathrm{N}_{\mathcal{G}}(v)$ by $v^{\perp}$ and we may write $v \perp w$ to denote that $v$ and $w$ are adjacent.

A subgraph of a graph $\mathcal{G}=(V, E)$ is a graph $\mathcal{H}=(W, F)$ such that $W \subseteq V$ and $F \subseteq E$. We call $\mathcal{H}$ induced if for any $v, w \in W$ the following holds: $\{v, w\}$ is an edge of $\mathcal{H}$ if and only if $\{v, w\}$ is an edge of $\mathcal{G}$. In that case $F$ is completely 
determined by $W$ and $E$.

A path in $\mathcal{G}$ is a sequence of vertices in which any two consecutive vertices are adjacent. The length of a path is the number of elements in the sequence minus one. We call $\mathcal{G}$ connected provided that, given any two vertices $v$ and $w$ of $\mathcal{G}$, there exists a path from $v$ to $w$. Define a binary relation $\equiv$ on $V$ as follows: $v \equiv w$ if and only if $v$ and $w$ are connected by a path. The relation $\equiv$ is obviously an equivalence relation. The connected components of $\mathcal{G}$ are the equivalence classes under the relation $\equiv$. The notion of path yields a natural notion of distance on $\mathcal{G}$ : given $v, w$ two vertices of $\mathcal{G}$, the distance $d_{\mathcal{G}}(v, w)=d(v, w)$ of $v$ and $w$ is the shortest integer $d$ such that there exists a path of length $d$ from $v$ to $w$. If $v$ and $w$ belong to different connected components of $\mathcal{G}$ we say that $d(v, w)=\infty$. The diameter of $\mathcal{G}$ from a vertex $v$ is the largest distance from $v$ to a vertex of $\mathcal{G}$. The diameter of $\mathcal{G}$ (without further reference to a vertex) is the largest diameter of $\mathcal{G}$ from some vertex.

An $s$-arc of $\mathcal{G}$ is an ordered $(s+1)$-tuple $\left(v_{0}, v_{1}, \ldots, v_{s}\right)$ such that $\left\{v_{i-1}, v_{i}\right\}$ is an edge of $\mathcal{G}$ for all $i \in\{1,2, \ldots, s\}$ and $v_{j-1} \neq v_{j+1}$ for all $j \in\{1, \ldots, s-1\}$.

A circuit in a graph $\mathcal{G}$ is a path $c=\left(v_{0}, \ldots, v_{n}, v_{0}\right)$ from a vertex to itself. The girth of $\mathcal{G}$ is the smallest integer $g$ such that there exists a circuit of length $g$ in $\mathcal{G}$. If there is no circuit in $\mathcal{G}$, we say that the girth of $\mathcal{G}$ is $\infty$.

We say that $\mathcal{G}=(V, E)$ is a complete graph provided that $E$ is the set of all pairs of distinct elements of $V$. It is common to denote a complete graph of $n$ vertices with $\mathcal{K}_{n}$. A synonym of complete graph is clique. If $\mathcal{K}$ is a subgraph of $\mathcal{G}$ and $\mathcal{K}$ is a complete graph, we prefer to say that $\mathcal{K}$ is a clique of $\mathcal{G}$ rather than a complete subgraph of $\mathcal{G}$.

We say that a property $P$ of a graph $\mathcal{G}$ is local provided that the induced subgraph on the neighborhood of every vertex has the property $P$. In particular, if $\mathcal{H}$ is a graph, we say that $\mathcal{G}$ is locally $\mathcal{H}$ if the subgraph induced on the neighborhood of any vertex of $\mathcal{G}$ is isomorphic to $\mathcal{H}$.

Let $\mathcal{G}=(V, E)$ be a graph and let $G$ be a group acting on $V$. This action induces an action of $G$ on pairs of elements of $V$ given by $g(\{v, w\})=$ $\{g(v), g(w)\}$. We say that $G$ acts on $\mathcal{G}$ provided that $G$ preserves the adjacency relation of $\mathcal{G}$, i.e. $g(e) \in E$ for any $e \in E$. In that case, a permutation of $G$ is called an automorphism of $\mathcal{G}$. The set of all automorphisms of $\mathcal{G}$ is endowed with a group structure and is called the automorphism group of $\mathcal{G}$, denoted by $\operatorname{Aut}(\mathcal{G})$. We say that $\mathcal{G}$ is vertex-transitive provided that $\operatorname{Aut}(\mathcal{G})$ has one orbit on the set of vertices of $\mathcal{G}$. We call $\mathcal{G}$ edge-transitive provided that $\operatorname{Aut}(\mathcal{G})$ acts transitively on the set of edges of $\mathcal{G}$. Observe however that vertex-transitivity and edge-transitivity do not imply each other.

Suppose that $\operatorname{Aut}(\mathcal{G})$ acts 2-transitively on the set of vertices of $\mathcal{G}$. Then 
obviously $E$ is empty or is the set of all pairs of distinct elements of $V$. In the latter case $\mathcal{G}$ is a complete graph. Hence $n$-transitivity is a concept that is not well suited to graphs. The concept of arc-transitivity may be preferred.

Let $G$ be a group of automorphisms of a graph $\mathcal{G}$ and let $s \geq 1$ be an integer. We say that $\mathcal{G}$ is $(G, s)$-arc-transitive provided that $G$ acts transitively on the arcs of $\mathcal{G}$ of length $s$. We say that $\mathcal{G}$ is $s$-arc-transitive provided that it is $(\operatorname{Aut}(\mathcal{G}), s)$ arc-transitive.

A 1-arc-transitive graph is also called arc-transitive for the sake of brevity. Observe that arc-transitivity does not force the graph to be empty nor complete.

\subsection{Incidence geometries}

Let $I$ be a finite set whose elements are called types while $I$ itself is called a set of types. A triple $\Gamma=(X, *, \tau)$ is called a pregeometry over $I$ if

1. $X$ is a set whose elements are called elements of $\Gamma$;

2. $*$ is a symmetric and reflexive relation on $X$ called the incidence relation of $\Gamma$;

3. $\tau$ is a mapping from $X$ to $I$, called the type function of $\Gamma$, such that distinct elements $x, y \in X$ with $x * y$ satisfy $\tau(x) \neq \tau(y)$.

If $\tau$ is surjective and if any maximal set of mutually incident elements of $\Gamma$ contains one element of each type then $\Gamma$ is called a geometry. The rank of $\Gamma$ is the cardinality of the set of types $I$.

A set of mutually incident elements is called a flag; if it contains one element of each type, it is called a chamber. A geometry is called flag-transitive if its automorphism group is transitive on the set of its chambers. The type of a flag is the set of types of the elements of the flag.

Let $\Gamma$ denote a geometry. The residue of a flag $F$ of $\Gamma$ is the set of all elements in $X \backslash F$ incident to every element in $F$ together with the incidence relation. It is an easy exercise to check that the residue of $F$ is a geometry.

For every flag $F$ of $\Gamma$, the $J$-shadow of $F$ is the set of all elements of type in $J \subseteq I$ that are incident with $F$. It inherits the incidence of $\Gamma$.

The truncation of $\Gamma$ on the set of types $J \subseteq I$ is the pregeometry ${ }_{J} \Gamma=$ $\left({ }_{J} X,{ }_{J} *{ }_{J} \tau\right)$ over $J$ such that ${ }_{J} X$ is the preimage $\tau^{-1}(J)$, and such that ${ }_{J} *$ (resp. ${ }_{J} \tau$ ) is the restriction of $*$ (resp. $\tau$ ) to ${ }_{J} X$. In other words, ${ }_{J} \Gamma$ is the restriction of $\Gamma$ on the elements of type is in $J$. 
A geometry is called residually connected provided that the incidence graph of the residue of any flag of rank at most $n-2$ is connected. A geometry is called firm provided that any flag of rank $n-1$ is contained in at least two chambers.

The incidence graph $(X, *)$ of $\Gamma$ is the graph whose vertices are elements of $X$ and adjacency is provided by incidence. By convention, if the graph is drawn, loops are deleted.

\subsection{Coset geometries}

According to Tits [34], given a group $G$ and a family of its subgroups $\left\{G_{0}, \ldots\right.$, $\left.G_{n-1}\right\}$, we define a pregeometry $\Gamma\left(G,\left\{G_{0}, \ldots, G_{n-1}\right\}\right)$ as follows. The type set of $\Gamma$ is the set $I=\{0, \ldots, n-1\}$; the elements of $\Gamma$ are the right cosets $G_{i} g$ for $i=0, \ldots, n-1$ and $g \in G$; incidence is defined by nonempty intersection, i.e. $G_{i} g * G_{j} h \Longleftrightarrow G_{i} g \cap G_{j} h \neq \emptyset$. If the pregeometry $\Gamma$ is flag-transitive then it is a geometry. The subgroups $G_{0}, \ldots, G_{n-1}$ are called maximal parabolic subgroups of $\Gamma$. Any intersection of maximal parabolic subgroups $\cap_{j \in J} G_{j}$ with $J \subset I$ is called a parabolic subgroup; the subgroups $\cap_{j \in I \backslash\{i\}} G_{j}$ are called minimal parabolic subgroups; the subgroup $\cap_{i \in I} G_{i}$ is called the Borel subgroup of $\Gamma$. A geometry arising in this way is called a coset geometry. A coset geometry $\Gamma$ is residually connected provided that each nonminimal parabolic subgroup of $\Gamma$ is generated by its proper parabolic subgroups.

\subsection{Diagram geometries}

A diagram of a (pre)geometry is a labelled graph providing information on its residues of rank 2 . Let $\Gamma=(X, *, t)$ be a pregeometry over some set of types $I$. The digon diagram associated to $\Gamma$ consists of a nonoriented graph of $|I|$ vertices named after the elements of $I$. Two vertices corresponding to the elements $i, j \in I$ are joined by an edge in the graph if the following two conditions hold:

1. there exists a flag $F$ of type $I \backslash\{i, j\}$ in $\Gamma$; and

2. there exist two elements of type $i$ and $j$ in $\Gamma_{F}$ which are not incident in $\Gamma_{F}$.

We say that a pregeometry belongs to its diagram.

Suppose from now on that $\Gamma=(X, *, \tau)$ is a firm, residually connected, flagtransitive geometry of rank 2 over the set of type $I=\{i, j\}$. It is possible to refine the digon diagram of $\Gamma$ with some parameters that provides further information about the residues and the flags of $\Gamma$. We say that $x, y \in X$ are at distance $k$ if they are at distance $k$ in the incidence graph $(X, *)$. For $j \in I$, the 


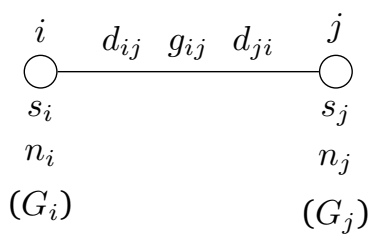

Figure 3: The Buekenhout diagram of a (coset) geometry

$j$-diameter $d_{j}$ of $\Gamma$ is the greatest number occuring as a diameter of $(X, *)$ for some element of type $j$. The diameter of $\Gamma$ is defined as $d:=\max \left\{d_{i} \mid i \in I\right\}$. The gonality is the smallest $\infty \geq g>0$ such that $(X, *)$ has a circuit of length $2 g$. Observe that every circuit in $(X, *)$ has even length. The order of an element $x$ of type $j$ is $s_{j}:=\left|\Gamma_{x}\right|-1$. That information is summed up in the diagram of Figure 3 where $n_{k}$ is the number of elements of type $k$ with $k=i, j$.

The Buekenhout diagram of $\Gamma$ is its digon diagram together with the parameters given in Figure 3. If $\Gamma$ is a coset geometry, we moreover provide stabilizers $G_{i}$ for all $i \in I$. More generally, if $\Gamma$ is a firm, residually connected, flag-transitive geometry of arbitrary rank, its Buekenhout diagram is its digon diagram together with parameters given as in Figure 3.

Sometimes $G_{i}$ acts with a kernel $K_{i} \triangleleft G_{i}$, in which case we write $G_{i}=$ $K_{i} \cdot\left(G_{i} / K_{i}\right)$ to emphasize the kernel of this action.

We use the following conventions: in case the labels of an edge are $d_{i}=g=$ $d_{j}=n$, we write only $n$ above the edge for any $i, j \in I$; in case $n=2$, we do not draw any edge at all; if $n=3$, we draw the edge without any label. More conventions are useful in some circumstances. Let us mention only two more. If $\left(d_{i j}, g_{i j}, d_{j i}\right)=(3,3,4), s_{i}=1$ and $s_{j}=n \geq 2$ then we write $\mathrm{C}$ over the stroke. In this case $\Gamma$ is a complete graph of $n+2$ vertices. The notation $\mathrm{C}$ is used to suggest the word 'circle' or 'complete graph'. If $\left(d_{i j}, g_{i j}, d_{j i}\right)=$ $(5,5,6)$ and $\left(s_{i}, s_{j}\right)=(1,2)$, we write $\mathrm{P}$ over the stroke instead because the corresponding residue is isomorphic to the rank 2 geometry of vertices and edges of the Petersen graph.

In an Atlas [27], Leemans provides diagram geometries for the nine smallest sporadic simple groups. In particular, he gives rank 4 geometries for the groups $\mathrm{M}_{11}$ and $\mathrm{J}_{1}$ over the diagrams of Figures 4 and 5. We prove in Theorem 9.4 that these two geometries are rank 4 residues of $\Gamma_{\mathrm{IvSh}}$.

Convention. When the context is not ambiguous, it is useful to adopt the convention that 'geometry' means 'firm, residually connected geometry'. 


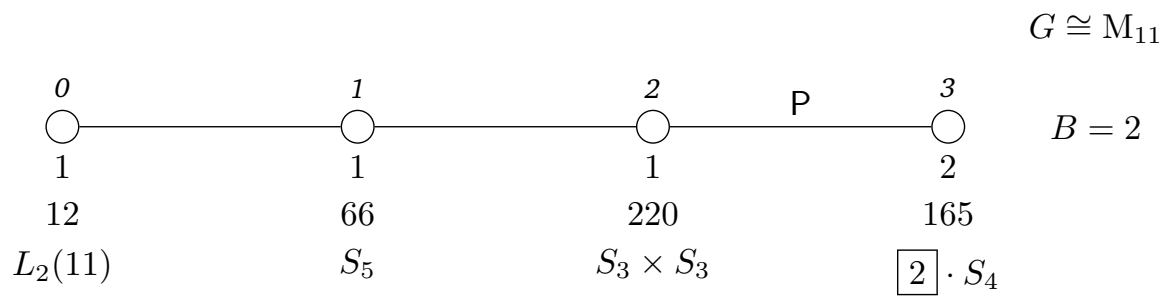

Figure 4: The diagram of the geometry $\Gamma_{\mathrm{M}_{11}}$

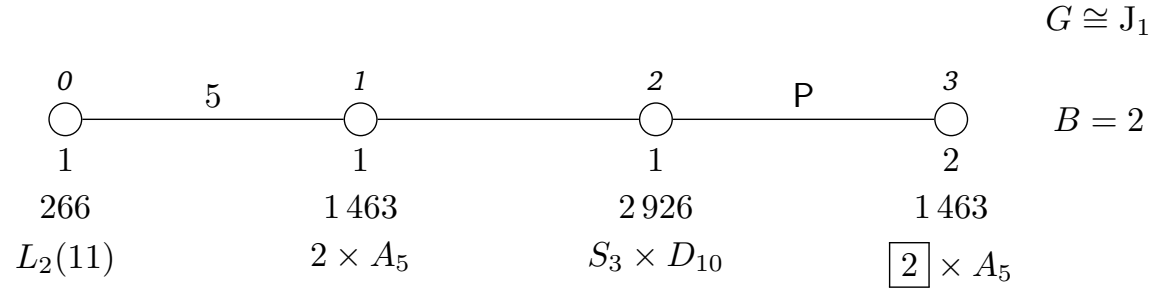

Figure 5: The diagram of $\Gamma_{J_{1}}$

\section{Lattices of subgroups}

In our paper, we make abundant use of lattices of subgroups in order to produce and describe coset geometries. Let $\Gamma=\Gamma\left(G,\left(G_{i}\right)_{i \in I}\right)$ be a firm, residually connected, flag-transitive coset geometry. The Borel subgroup $B=\cap_{i \in I} G_{i}$ of $\Gamma$ is the stabilizer of some chamber $C$ of $\Gamma$. The maximal parabolic subgroup $G_{i}, i \in I$ of $\Gamma$ is the stabilizer of the $i$-element of $C$. Accordingly, $G_{J}, J \subseteq I$ is the stabilizer of the flag $F \subseteq C$ of type $J$. The collection of all parabolic subgroups $G_{J}, J \subseteq I$ of $\Gamma$ structured by inclusion yields a boolean lattice $L$ of subgroups of $G$. However, notice that not every boolean lattice of subgroups of a group $G$ yields a geometry. Lemma 3.1 provides a characterization of residual connectedness in coset geometries.

Lemma 3.1. A coset geometry $\Gamma$ is residually connected if and only if every nonminimal parabolic subgroup of $\Gamma$ is generated by the parabolic subgroups it contains properly.

Proof. See [10, Lemma 1.8.9].

Lemma 3.1 motivates the introduction of the following terminology. A boolean lattice of subgroups $L$ of some group $G$ is called generating provided that every nonminimal subgroup in $L$ is generated by the subgroups in $L$ that it 
contains properly. Obviously a residually connected coset geometry yields a generating boolean lattice of parabolic subgroups.

As a matter of fact, we rely deeply on the maximal subgroups of $\mathrm{O}^{\prime} \mathrm{N}$. The classification of those is due to Wilson [36], Yoshiara [37] (computer-free proof) and Ivanov, Tsaranov \& Shpectorov [25] independently. See also Soicher [32].

In [26], Leemans built an algorithm that computes the subgroup lattice of a permutation group $G$ based on the following ideas. Given a permutation group $(G, \Omega)$ and a construction of the classes of the maximal subgroups of $G$ with representatives $M_{1}, M_{2}, \ldots, M_{n}$, Leemans looks for a faithful permutation representation of minimal degree for every $M_{i}, i \in\{1, \ldots, n\}$ which is an ordered pair $\left(M_{i}, M_{i j}\right)$ where $M_{i j}$ is a proper subgroup of $M_{i}$ of largest order containing no normal proper subgroup of $M_{i}$. From this on it is assumed (and all right for $G=\mathrm{O}^{\prime} \mathrm{N}$ ) that the maximal subgroups of each maximal subgroup of $G$ are computable. Afterwards a delicate analysis of overlappings between $\Sigma_{i}$ and every other $\Sigma_{j}$ occurs.

Leemans implemented this algorithm in MAGMA and managed to compute the subgroup lattice of $\mathrm{O}^{\prime} \mathrm{N}$. This paper has been extended in a work by Connor and Leemans which now contains subgroup lattices of many finite almost simple groups, including $\mathrm{O}^{\prime} \mathrm{N}$ and $\mathrm{O}^{\prime} \mathrm{N}: 2$ [16].

\begin{tabular}{||c|c|c|c|l|l||}
\hline Nr. & Structure & Order & Length & Maximal Subgroups & Minimal Overgroups \\
\hline 1 & $A_{5}$ & 60 & 1 & $2(5), 3(6), 4(10)$ & \\
\hline 2 & $A_{4}$ & 12 & 5 & $6,7(4)$ & 1 \\
\hline 3 & $D_{10}$ & 10 & 6 & $5,8(5)$ & 1 \\
\hline 4 & $S_{3}$ & 6 & 10 & $7,8(3)$ & 1 \\
\hline 5 & 5 & 5 & 6 & 9 & 3 \\
\hline 6 & $2^{2}$ & 4 & 5 & $8(3)$ & 2 \\
\hline 7 & 3 & 3 & 10 & 9 & $2(2), 4$ \\
\hline 8 & 2 & 2 & 15 & 9 & $3(2), 4(2), 6$ \\
\hline 9 & 1 & 1 & 1 & & $5(6), 7(10), 8(15)$ \\
\hline
\end{tabular}

Table 1: The subgroup lattice of $A_{5}$

Table 1 gives an example for the alternating group $A_{5}$. Each conjugacy class of subgroups is listed with a number $c$, a structure describing the subgroups of the class, the order of the groups in this class, the length of the class and the labels of the conjugacy classes of subgroups where the maximal subgroups and minimal overgroups are. In the antepenultimate (resp. last) column, when a class number $x$ is followed by a number between parentheses, this number $n$ means that there are $n$ subgroups (resp. overgroups) of conjugacy class $\# x$ contained (resp. containing) a given group of the class \#c. For instance, class 
labelled \#6 contains 5 Klein 4-groups in Table 1. Each Klein 4-group of that class has exactly 3 maximal subgroups in class \#8, that are cyclic groups of order 2 . Moreover, each of these cyclic groups of order 2 is a maximal subgroup of two groups of class \#3, two groups of class \#4 and one group of class \#6.

The proof of our main result is based on the identification of boolean lattices in the subgroup lattice of various groups, including $\mathrm{M}_{11}, \mathrm{~J}_{1}$ and $\mathrm{O}^{\prime} \mathrm{N}$, that yield coset geometries, as well as on observations in the subgroup lattice of $\mathrm{O}^{\prime} \mathrm{N}: 2$. We denote the subgroup lattice available in [26] of $\mathrm{O}^{\prime} \mathrm{N}$ by $\Lambda_{\mathrm{O}^{\prime} \mathrm{N}}$; the subgroup lattice of $\mathrm{O}^{\prime} \mathrm{N}: 2$ available in [16] is $\Lambda_{\mathrm{O}^{\prime} \mathrm{N}: 2}$. When we refer to 'a subgroup of class $\# x$ in $\Lambda_{\mathrm{O}^{\prime} \mathrm{N}: 2}$ ', we mean some subgroup of the conjugacy class of subgroups labelled $\# x$, as given in our references. We always make clear whether we deal with $\Lambda_{\mathrm{O}^{\prime} \mathrm{N}}$ or $\Lambda_{\mathrm{O}^{\prime} \mathrm{N}: 2}$. We often draw boolean lattices or partial subgroup lattices that are extracted from $\Lambda_{\mathrm{O}^{\prime} \mathrm{N}}$ or $\Lambda_{\mathrm{O}^{\prime} \mathrm{N}: 2}$ in order to help the reader. We do not prove their correctness because it consists only of an observation of the lattices $\Lambda_{\mathrm{O}^{\prime} \mathrm{N}}$ and $\Lambda_{\mathrm{O}^{\prime} \mathrm{N}: 2}$.

\section{Two geometries for $M_{11}$ and $J_{1}$}

Among the maximal subgroups of $\mathrm{O}^{\prime} \mathrm{N}$ there are two sporadic groups (up to automorphism) namely the Janko group $\mathrm{J}_{1}$ and the Mathieu group $\mathrm{M}_{11}$. All subgroups of $\mathrm{O}^{\prime} \mathrm{N}$ isomorphic to $\mathrm{J}_{1}$ are conjugate while there are two conjugacy classes of subgroups isomorphic to $\mathrm{M}_{11}$. Those two classes are fused in $\operatorname{Aut}\left(\mathrm{O}^{\prime} \mathrm{N}\right)$. These groups and the related geometries of rank 4 that we are about to describe are crucial for the rest of this paper. Both of them possess a residue which is a rank 3 geometry with $L_{2}(11)$ acting flag-transitively. We start with the description of the latter geometry.

\subsection{A locally Petersen geometry with $L_{2}(11)$ acting flag-transi- tively}

The classification of locally Petersen graphs is due to Jonathan Hall [20]. There are exactly three such graphs. They are flag-transitive and actually distancetransitive. We describe them briefly with a further reference to Weisstein [35] and to Brouwer-Cohen-Neumaier [4].

1. The Conway-Smith graph of 63 vertices and 315 edges whose group of automorphisms is $3 \cdot S_{7}$. It is a triple cover of the next graph.

2. The graph of the 21 pairs of elements in $\{1, \ldots, 7\}$ such that two distinct pairs are adjacent provided they have nonempty intersection. It is 


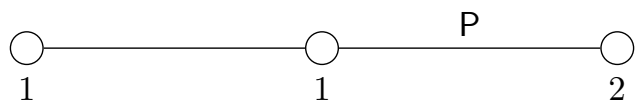

Figure 6: Diagram $\mathrm{A}_{2} \mathrm{P}$ of a locally Petersen geometry

the unique distance regular graph with 21 vertices and intersection array $[10,6 ; 1,6]$. Its automorphism group is $S_{7}$. It is the $(7,2)$-Kneser graph.

3. The Doro-Hall graph of 65 vertices and 325 edges whose automorphism group is $\mathrm{P} \Sigma \mathrm{L}(2,25)$.

It is immediate to see that each of those three graphs provides a geometry over the diagram $\mathrm{A}_{2} \mathrm{P}$ of Figure 6 whose 2-elements (planes) are the 3-cliques in the graph.

Conversely, Shpectorov [31] proved that there are exactly four flag-transitive geometries that belong to the diagram of Figure 6: the three locally Petersen graphs and a geometry built from the action of $L_{2}(11)$ on the short Galois line (see also [7, Section 9, geometry 3]). This terminology was used in [9] to denote the 2-transitive action of $L_{2}(11)$ on 11 points, as observed by Galois. We denote this geometry with $\Gamma_{L_{2}(11)}$ and we describe it further. Let us call elements of $\Gamma_{L_{2}(11)}$ points, lines and planes, by reading the diagram of Figure 6 from left to right. The diagram shows that there are 10 lines through each point and 2 points on each line. Therefore the truncation of $\Gamma_{L_{2}(11)}$ on its points and lines is a complete graph. The group $L_{2}(11)$ has two orbits on the trios of points: one of length 55 and the other of length 110 . The planes of $\Gamma_{L_{2}(11)}$ are the trios of the smallest orbit. Incidence is provided by inclusion.

\subsection{A rank 4 geometry for $M_{11}$}

Let us consider the problem of determining the flag-transitive geometries that are 'locally $\Gamma_{L_{2}(11)}$ '. In other words, what are the firm, residually connected, flag-transitive geometries over the diagram $A_{3} P$ where we assume that the stabilizer of a point is isomorphic to $L_{2}(11)$ (see Figure 7)? Let $\Upsilon$ be such a geometry with flag-transitive automorphism group $G$. We call elements of $\Upsilon$ points, lines, planes and hyperplanes by reading the diagram from left to right. The residue in $\Upsilon$ of every point is isomorphic to the geometry $\Gamma_{L_{2}(11)}$ described in Section 4.1, i.e. every point is on 11 lines. The diagram $A_{3} P$ shows that every line contains 2 points, hence the truncation of $\Upsilon$ on its points and lines is a graph $\mathcal{G}$ in which every point has 11 neighbors. The stabilizer of a point acts 2transitively on those 11 points, by assumption. Therefore the induced subgraph 


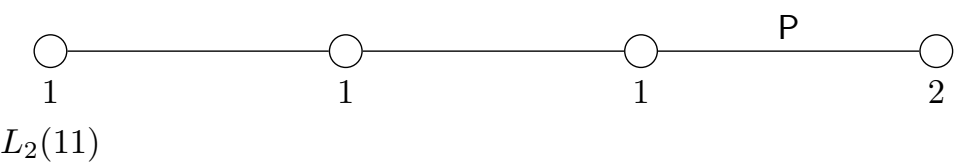

Figure 7: Diagram $A_{3} P$ with a residue isomorphic to $\Gamma_{L_{2}(11)}$

on the neighborhood $p^{\perp}$ of a point $p$ in $\mathcal{G}$ is either a complete graph or an empty graph. Moreover we read from the diagram that the residue of a plane of $\Upsilon$ contains 3 points and 3 lines forming a triangle. Since there are cycles of length 3 in $\mathcal{G}$, we conclude that $p^{\perp}$ is a complete graph. Therefore a point $p$ of $\mathcal{G}$ together with its neighborhood $p^{\perp}$ is a clique of 12 points (in which we observe trivially that every point has 11 neighbors). By residual connectedness of $\Upsilon, \mathcal{G}$ cannot be a disjoint union of 12-cliques. In conclusion $\Upsilon$ has 12 points on which $G$ must act transitively and on which the stabilizer of a point acts 2 -transitively, i.e. $G$ is 3 -transitive. This group must be of order $12 \times 660=7920$, i.e. $G$ is a primitive group on 12 points of order 7920 . We conclude that $G \cong \mathrm{M}_{11}$ (see [17] and [18, Chapter 6]).

We will denote the geometry $\Upsilon$ by $\Gamma_{\mathrm{M}_{11}}$ and we describe it further. Define the triple $\left(X_{\mathrm{M}_{11}}, *_{\mathrm{M}_{11}}, \tau_{\mathrm{M}_{11}}\right)$ as follows. The set $X_{\mathrm{M}_{11}}$ of varieties is the union of four sets of subsets of vertices of a 12-clique $K_{12}$ : the set of vertices of $K_{12}$, the set of pairs of vertices of $K_{12}$, the set of trios of vertices of $K_{12}$ and a set of 4-cliques of $K_{12}$. The latter set consists of one of the two orbits of 4-cliques occuring under the action of $\mathrm{M}_{11}$ on $K_{12}$, namely the orbit of cardinality 165 (while the second orbit has cardinality 330). The incidence $*_{\mathrm{M}_{11}}$ is defined by symmetrized inclusion in $K_{12}$ and the type function $\tau_{\mathrm{M}_{11}}$ associates the type $0,1,2,3$ to a vertex, pair of vertices, triple of vertices and 4-cliques of $X_{\mathrm{M}_{11}}$ respectively. Then $\Gamma_{\mathrm{M}_{11}}=\left(X_{\mathrm{M}_{11}}, *_{\mathrm{M}_{11}}, \tau_{\mathrm{M}_{11}}\right)$ is a firm, residually connected, flag-transitive geometry over $\{0,1,2,3\}$ under the action of $\mathrm{M}_{11}$.

This proves existence and uniqueness of a firm, residually connected, flagtransitive geometry over the diagram $\mathrm{A}_{3} \mathrm{P}$ with point stabilizer $L_{2}(11)$.

The geometry $\Gamma_{\mathrm{M}_{11}}$ is well known: it corresponds to Geometry 89 in [6] and Geometry 4.3 in [27]. We give its Buekenhout diagram in Figure 4.

\subsection{A rank 4 geometry for $\mathrm{J}_{1}$}

In this section we provide an existence and uniqueness proof of a flag-transitive geometry with automorphism group isomorphic to $\mathrm{J}_{1}$ over the diagram of Figure 8. We call this diagram $\mathrm{H}_{3}^{*} \mathrm{P}$ because it is the linear amalgam of a 


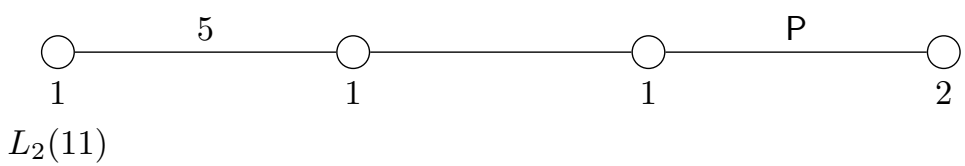

Figure 8: Diagram $\mathrm{H}_{3}^{*} \mathrm{P}$ with a residue isomorphic to $\Gamma_{L_{2}(11)}$

reversed Coxeter diagram $\mathrm{H}_{3}$ and the Petersen diagram $\mathrm{P}$.

Let us briefly consider the Livingstone graph $\mathcal{L}=\left(V_{\mathcal{L}}, E_{\mathcal{L}}\right)$ defined in [28] and further studied for instance in $[4,22,35]$. It is a distance regular graph of degree 11 with 266 vertices and 1463 edges. The automorphism group of $\mathcal{L}$ is isomorphic to $\mathrm{J}_{1}$. The stabilizer in $\mathrm{J}_{1}$ of a vertex is isomorphic to $L_{2}(11)$ and the stabilizer of an edge is isomorphic to $2 \times A_{5}$. We recall without proof some properties of the Livingstone graph. The first is proven in [4], the remaining ones are proven in [22].

Lemma $4.1([4,22])$. The Livingstone graph $\mathcal{L}$ is endowed with the following properties.

- $\mathcal{L}$ is distance-transitive.

- There are two orbits of pentagons in $\mathcal{L}$, say an orbit of white pentagons and an orbit of orange pentagons. The orbit of white pentagons is of size 2926 and the orbit of orange pentagons is of size 8778. The stabilizer of a white pentagon is isomorphic to $S_{3} \times D_{10}$ and the stabilizer of an orange pentagon is isomorphic to $2 \times D_{10}$.

- The stabilizer of a Petersen subgraph of $\mathcal{L}$ is isomorphic to $2 \times A_{5}$ and $\mathrm{J}_{1}$ has one orbit on the set of Petersen subgraphs of $\mathcal{L}$.

- Each white pentagon $P$ belongs to three Petersen subgraphs of $\mathcal{L}$. Each orange pentagon belongs to one Petersen subgraph of $\mathcal{L}$.

- The pointwise stabilizer of a white pentagon $P$ is isomorphic to $S_{3}$ and it permutes transitively the three Petersen subgraphs containing $P$.

We make use of Lemma 4.1 to define a geometry $\Gamma_{J_{1}}=\left(X_{J_{1}}, *_{J_{1}}, \tau_{J_{1}}\right)$ over the set of types $I_{\mathrm{J}_{1}}=\{0,1,2,3\}$. In Section 9.3, these types are rather named $4,1,3, h$ respectively where $h$ stands for 'hyperline' as defined in Section 10. The 0 -elements are the vertices of $\mathcal{L}$, the 1 -elements are the edges of $\mathcal{L}$, the 2-elements are the white pentagons of $\mathcal{L}$ and the 3-elements are the Petersen subgraphs of $\mathcal{L}$. The incidence relation is induced by inclusion. 
Theorem 4.2. There exists a unique flag-transitive geometry over the diagram of Figure 8 with automorphism group isomorphic to $\mathrm{J}_{1}$. It is geometry $\Gamma_{\mathrm{J}_{1}}$.

Proof. We divide the proof of this Theorem in two parts: existence is proven in Section 4.3.1 and uniqueness in Section 4.3.2.

This geometry was first observed by Buekenhout in 1980 but he did not publish it. In [24], Ivanov and Shpectorov implicitly assert the existence of this geometry. It is coming as a residue of their rank 5 geometry for the group $\mathrm{O}^{\prime} \mathrm{N}$. The geometry $\Gamma_{J_{1}}$ is Geometry 4.1 for $J_{1}$ in Leemans [27]. Moreover Leemans and Gottschalk obtained the uniqueness of that geometry under the further hypotheses that the geometry is residually weakly primitive [19]. Notice furthermore that Perkel [30] used a rank 3 geometry obtained by Buekenhout in [5] to characterize the Livingstone graph and the Janko group $\mathrm{J}_{1}$. The geometry he used is Geometry 28 in [6] and is a truncation of $\Gamma_{J_{1}}$ on the set of types $\{0,1,2\}$.

\subsubsection{Existence proof}

The triple $\Gamma_{\mathrm{J}_{1}}=\left(X_{\mathrm{J}_{1}}, *_{\mathrm{J}_{1}}, \tau_{\mathrm{J}_{1}}\right)$ is a pregeometry according to Section 2.2. It is actually a geometry because any maximal flag contains one element of each type, by construction. Moreover $J_{1}$ acts as a group of automorphisms on $\Gamma_{J_{1}}$. We now prove that this action is flag-transitive. Let $F=\{v, e, p, h\}$ and $F^{\prime}=$ $\left\{v^{\prime}, e^{\prime}, p^{\prime}, h^{\prime}\right\}$ be two chambers of $\Gamma_{\mathrm{J}_{1}}$, where $v$ and $v^{\prime}$ are vertices, $e$ and $e^{\prime}$ are edges, $p$ and $p^{\prime}$ are pentagons and $h$ and $h^{\prime}$ are Petersen subgraphs.

Since $\mathcal{L}$ is distance-transitive, there exists an element $\gamma$ of $\mathrm{J}_{1}$ mapping $\{v, e\}$ onto $\{\gamma(v), \gamma(e)\}=\left\{v^{\prime}, e^{\prime}\right\}$. The elements $\gamma(p), \gamma(h)$ are incident and are incident with both $v^{\prime}$ and $e^{\prime}$. It remains to show that there exists $\delta \in \operatorname{Stab}_{J_{1}}\left\{v^{\prime}, e^{\prime}\right\}$ mapping $\{\gamma(p), \gamma(h)\}$ onto $\{\delta(\gamma(p)), \delta(\gamma(h))\}=\left\{p^{\prime}, h^{\prime}\right\}$. The stabilizer of $\left\{v^{\prime}, e^{\prime}\right\}$ is the pointwise stabilizer of two adjacent vertices $v^{\prime}, w$ in $\mathcal{L}$ (where $e^{\prime}=\left\{v^{\prime}, w\right\}$ ). The 2-arc-transitivity of $\mathcal{L}$ implies that this stabilizer acts transitively on the neighbors of $w$ that are at distance 2 from $v^{\prime}$, hence it acts transitively on the set of white pentagons containing $v^{\prime}$ and $w$. Those pentagons are precisely the white pentagons incident to $\left\{v^{\prime}, e^{\prime}\right\}$. There are now three Petersen subgraphs incident with $\left\{v^{\prime}, e^{\prime}, p^{\prime}\right\}$. By Lemma 4.1, $\operatorname{Stab}_{J_{1}}\left\{v^{\prime}, e^{\prime}, p^{\prime}\right\} \cong S_{3}$ permutes transitively those three Petersen subgraphs. This achieves the proof that $J_{1}$ acts flag-transitively on $\Gamma_{\mathrm{J}_{1}}$ : we showed that given any two chambers $F$ and $F^{\prime}$ there exists an element of $\mathrm{J}_{1}$ mapping $F$ onto $F^{\prime}$.

Firmness is obvious from the construction. In view of the fact that $\mathcal{L}$ is a connected graph and that this also holds for the incidence graph of $\Gamma_{J_{1}}$, there readily follows that $\Gamma_{J_{1}}$ is residually connected. It is also an easy exercise to show that the Buekenhout diagram of $\Gamma_{J_{1}}$ is as depicted in Figure 5: it is enough 
to observe that the residue of a point is the geometry of Section 4.1 and that the residue of a Petersen subgraph is the geometry of a hemidodecahedron (quotient of a dodecahedron by the central symmetry).

\subsubsection{Uniqueness proof}

As for uniqueness, assume that $\Gamma$ is a flag-transitive geometry over the diagram $\mathrm{H}_{3}^{*} \mathrm{P}$ admitting a flag-transitive action of $\mathrm{J}_{1}$. That geometry must have $266=\left|J_{1}\right| /\left|L_{2}(11)\right|$ points. The diagram shows that the residue of a line has 2 points. Since we also know that the residue of a point is the flag-transitive $A_{2} P$ geometry described in Section 4.1, every point is incident to 11 lines. Hence there are $1463=\frac{266 \times 11}{2}$ lines. The truncation of $\Gamma$ on the 0 - and 1-elements is thus a graph $\mathcal{G}$, with incidence provided by inclusion. Since we assumed that $\mathrm{J}_{1}$ acts on $\Gamma$, this is already enough to conclude that this truncation is the Livingstone graph $\mathcal{L}$. Then we can count the number of 2-elements: there are 55 2-elements in the residue of a point and there are 5 points in the residue of a 2-element, that we now call pentagons. Therefore there are $\frac{266 \times 55}{5}=2926$ pentagons in $\Gamma$. Now the diagram of the residue of a 3-element is the diagram of only two flag-transitive geometries, necessarily finite: they are the geometries of a dodecahedron and a hemidodecahedron. If it were a dodecahedron, there would be $\frac{266 \times 55}{20}=\frac{1463}{2} 3$-elements, a contradiction. Hence it is a hemidodecahedron and there are 1463 hemidodecahedra in $\Gamma$. There are now several ways to conclude. Recall that the truncation on vertices and edges of a hemidodecahedron is a Petersen graph. Hence hemidodecahedra of $\Gamma$ are Petersen subgraphs of $\mathcal{L}$. Since there are exactly 1463 Petersen subgraphs in $\mathcal{L}$ (see for instance [22, Section 3.1]), they are the 3-elements of $\Gamma$. The same methods show that pentagons of $\Gamma$ are the white pentagons of Lemma 4.1. This amounts to the construction of $\Gamma_{J_{1}}$ and finishes the uniqueness proof.

\subsection{Boolean lattices of $\Gamma_{\mathrm{M}_{11}}$ and $\Gamma_{\mathrm{J}_{1}}$}

In Sections 4.2 and 4.3 we established existence and uniqueness of two flagtransitive geometries. Both of them come with a group and can be seen as coset geometries as defined in Section 2.3. The purpose of this section is to compute the boolean lattice of parabolic subgroups of each of them. Let us start with $\Gamma_{\mathrm{M}_{11}}$.

The flag-transitive geometry $\Gamma_{\mathrm{M}_{11}}$ constructed in Section 4.2 can be seen as a coset geometry $\Gamma\left(\mathrm{M}_{11},\left\{G_{0}, G_{1}, G_{2}, G_{3}\right\}\right)$. We now identify the boolean lattice of subgroups of $\Gamma_{\mathrm{M}_{11}}$ in the subgroup lattice $\Lambda_{\mathrm{M}_{11}}$ of $\mathrm{M}_{11}$ given in Table 4 (this lattice is borrowed from [16]). We do this by applying and describing 
Tits's algorithm. We provide much detail because it is a description of a general method that permits to 'see' a geometry inside a lattice of subgroups.

Theorem 4.3. The boolean lattice $L_{\mathrm{M}_{11}}$ of the geometry $\Gamma_{\mathrm{M}_{11}}$ is depicted in Figure 9. Moreover there exists a unique such boolean lattice of subgroups in $\mathrm{M}_{11}$ up to conjugacy.

Proof. By construction, the stabilizer of a point is isomorphic to $L_{2}(11)$. The 12 subgroups of conjugacy class $\# 3$ are in bijection with the points of $\Gamma_{\mathrm{M}_{11}}$. Since all subgroups of $\mathrm{M}_{11}$ isomorphic to $L_{2}(11)$ are conjugate, all choices of a particular subgroup $L_{2}(11)$ are equivalent. Let $G_{0} \cong L_{2}(11)$ be a subgroup of class \#3. The stabilizer of an edge $G_{1}$ is isomorphic to $S_{5}$ because the residue of an edge in $\Gamma_{\mathrm{M}_{11}}$ is the direct sum of a Petersen graph and a rank 1 geometry of 2 points. The 120 subgroups of conjugacy class \#6 are also in bijection with the edges of $\Gamma_{\mathrm{M}_{11}}$. Notice however that not all choices of subgroups of class $\# 6$ are equivalent at this stage. Indeed a point is incident to 11 edges and an edge is incident to two points. The stabilizer of a flag point-edge is of order $60=\frac{660}{11}=\frac{120}{2}$. Thus $G_{01}=G_{0} \cap G_{1}$ is a subgroup of class \#10: the lattice $\Lambda_{\mathrm{M}_{11}}$ of Table 4 shows that every subgroup of class \#10 (isomorphic to $A_{5}$ ) sits in two subgroups of class \#3 (stabilizers of points) and one subgroup of \#6 (stabilizers of edges). On the other hand, every subgroup of class \#3 contains 11 subgroups of class \#10 and every subgroup of class \#6 contains one subgroup of class \#10. It should be clear at this point that $G_{1}$ can be chosen inside class \#6 in such a way that $G_{0} \cap G_{1}$ is a subgroup $G_{01}$ of class \#10. Moreover, notice that the coset geometry $\Gamma\left(\mathrm{M}_{11},\left\{G_{0}, G_{1}\right\}\right)$ is the geometry of a complete graph on 12 points, i.e. the truncation of $\Gamma_{\mathrm{M}_{11}}$ on the set of types $\{0,1\}$.

Every flag point-edge can be extended in 10 flags point-edge-trio. The parabolic subgroup $G_{01}$ (of class \#10) contains 10 subgroups of class \#31. Hence we see that $G_{0} \cap G_{1} \cap G_{2}=G_{012}$ is a subgroup of class \#31. Now $G_{02}$ and $G_{12}$ must be two distinct subgroups of class \#23 (isomorphic to $D_{12} \cong 2 \times S_{3}$ ). Indeed the diagram shows that every flag point-trio lies in two flags point-edge-trio and that every flag edge-trio lies in two flags point-edge-trio. Hence $G_{02}$ and $G_{12}$ have to be twice larger than $G_{012}$. The lattice $\Lambda_{\mathrm{M}_{11}}$ shows that the only choice for $G_{02}$ and $G_{12}$ is to take distinct subgroups of class \#23, which is possible because every subgroup of class \#31 sits in three subgroups of class \#23. Notice furthermore that $G_{02}$ and $G_{12}$ must be distinct for otherwise $G_{02} \cap G_{12}=G_{02}=G_{12}$ and the geometry would not be firm. The parabolic subgroup $G_{2}$ must contain subgroups of class \#23 and there must be 110 subgroups conjugate to $G_{2}$. Indeed, there are 110 trios in $\Gamma_{\mathrm{M}_{11}}$ and $\mathrm{M}_{11}$ permutes them transitively. Quick inspection of the overgroups of a subgroup of class \#23 shows that $G_{2}$ is a subgroup of class \#16, isomorphic to $S_{3} \times S_{3}$. 


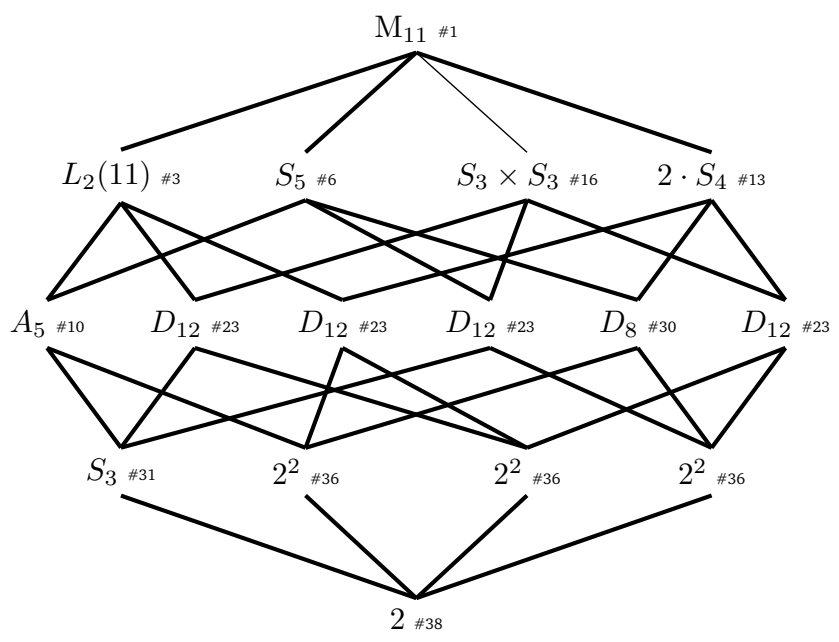

Figure 9: The boolean lattice of $\Gamma_{\mathrm{M}_{11}}$ identified in $\Lambda_{\mathrm{M}_{11}}$

The residue of a flag point-edge is isomorphic to the geometry of a Petersen graph. Moreover $G_{01} \cong A_{5}$ acts flag-transitively on this residue. The stabilizer of a point in this action is isomorphic to $S_{3}$, the stabilizer of an edge is isomorphic to $2^{2}$, and the Borel subgroup (i.e. the stabilizer of a chamber) is a cyclic group of order 2 (see for instance [11]). Hence $G_{012} \cong S_{3}$ must be a subgroup of class \#31 (which we already know) and $G_{013} \cong 2^{2}$ must be a subgroup of class \#36, the only conjugacy class of subgroups isomorphic to $2^{2}$ in $\mathrm{M}_{11}$. The Borel subgroup of $\Gamma_{\mathrm{M}_{11}}$ is a subgroup of class \#39.

At last, we come to $G_{3}$. The residue of a 3-element of $\Gamma_{\mathrm{M}_{11}}$ is the geometry of a tetrahedron. Hence $\left|G_{3}\right|$ has order divisible by 24 . There are 55 elements of type 3 through every point, and there are 4 points through every 3 -element. Therefore there are $165=\frac{12 \times 55}{4}$ elements of type 3 in $\Gamma_{\mathrm{M}_{11}}$. Now $\frac{7920}{165}=48$ is the order of $G_{3}$. It is then clear that $G_{3}$ belongs to class \#13. This parabolic subgroup acts with a kernel of size 2 .

It is now an easy exercise to determine the remaining unknowns.

We provide the boolean lattice of $\Gamma_{\mathrm{M}_{11}}$ in Figure 9 where thick lines represent maximal intersection. We also provide the name of parabolic subgroups with the label of the conjugacy class to which the corresponding parabolic subgroup belongs in $\Lambda_{\mathrm{M}_{11}}$.

Theorem 4.4. The boolean lattice $L_{\mathrm{J}_{1}}$ of the geometry $\Gamma_{\mathrm{J}_{1}}$ is depicted in Figure 10. Moreover there exists a unique such boolean lattice of subgroups in $\mathrm{J}_{1}$ up to conjugacy. 


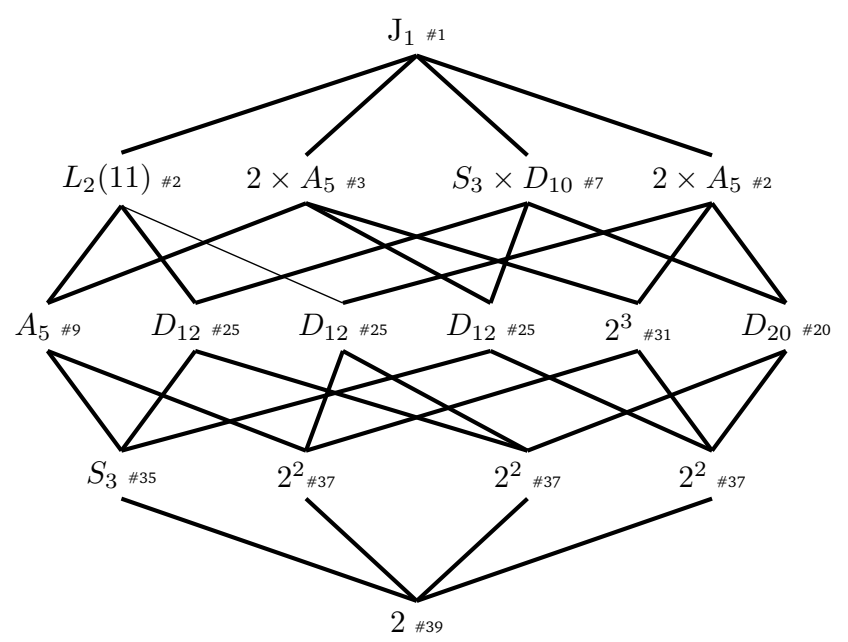

Figure 10: The boolean lattice of $\Gamma_{J_{1}}$ identified in $\Lambda_{J_{1}}$

Proof. The very same techniques described in the case of $\Gamma_{\mathrm{M}_{11}}$ can be used to compute and identify the boolean lattice of $\Gamma_{J_{1}}$ in the subgroup lattice $\Lambda_{J_{1}}$ of $\mathrm{J}_{1}$ given in Table 5 (borrowed from [16]). We provide the boolean lattice in Figure 10 (see also [19]).

\section{The rank 5 geometry of Ivanov-Shpectorov for $\mathrm{O}^{\prime} \mathrm{N}$}

Ivanov and Shpectorov give evidence for the existence of a geometry of rank 5 with $\mathrm{O}^{\prime} \mathrm{N}$ acting in [24]. Their starting point is the original paper of O'Nan that predicts and describes $\mathrm{O}^{\prime} \mathrm{N}$ [29]. Here is a sketch of their construction.

The starting point is the permutation representation of $\mathrm{O}^{\prime} \mathrm{N}$ on the (left) cosets of $J_{1}$. Using the fact that $J_{1}$ centralizes an involution of $\operatorname{Aut}\left(\mathrm{O}^{\prime} \mathrm{N}\right) \backslash \mathrm{O}^{\prime} \mathrm{N}$, they build a vertex-transitive graph $\mathcal{G}_{\text {IVSh }}$ of valency 1463 whose points are the cosets of $\mathrm{J}_{1}$ in $\mathrm{O}^{\prime} \mathrm{N}$ and in which the stabilizer of an edge is isomorphic to $2 \times S_{5}$. Vertices (resp. edges) of $\mathcal{G}_{\text {IvSh }}$ are the 0-elements (resp. 1-elements) of the geometry (with respect to our notation as in Figure 1). By looking at normalizers in $\mathrm{O}^{\prime} \mathrm{N}$ of subgroups isomorphic to $S_{3}$ lying in $\mathrm{J}_{1}$, they exhibit cliques of 6 vertices stabilized by subgroups of $\mathrm{O}^{\prime} \mathrm{N}$ isomorphic to $S_{3} \times A_{5}$. Those subgraphs are the 3 -elements. Then they consider the action of $L_{2}(11)<\mathrm{J}_{1}$ on $\mathcal{G}_{\mathrm{IvSh}}$ and after a few observations, they show that subgroups of $\mathrm{O}^{\prime} \mathrm{N}$ isomorphic to $\mathrm{M}_{11}$ 


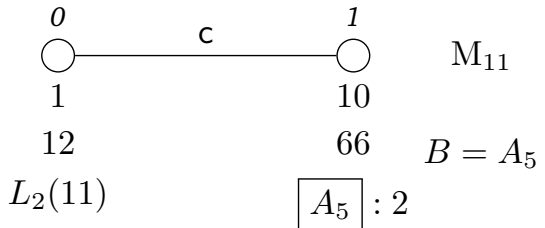

(a) $\mathrm{M}_{11}$ on 12 points

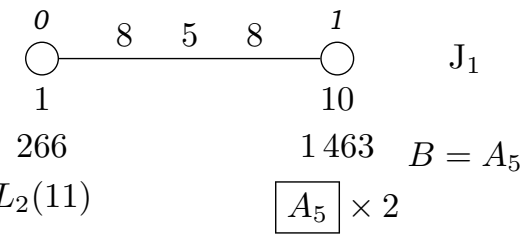

(b) The Livingstone graph

Figure 11: Rank 2 coset geometries for $\mathrm{M}_{11}$ and $\mathrm{J}_{1}$

stabilize cliques on 12-points in $\mathcal{G}_{\text {IvSh }}$. Those cliques are the 4-elements. Finally they look at involutions of $\mathrm{O}^{\prime} \mathrm{N}$ lying in subgroups isomorphic to $S_{3}<\mathrm{J}_{1}$. They consider the action of the centralizer of an involution in $\mathrm{O}^{\prime} \mathrm{N}$ on $\mathcal{G}_{\mathrm{IvSh}}$. This allows them to identify subgraphs of size 16 which in turn provide them with subgraphs that become the $h$-elements of the geometry. However the stabilizer of an $h$-element in $\mathrm{O}^{\prime} \mathrm{N}$ is not made explicit.

Ivanov and Shpectorov conclude their paper with the assertion that the elements they have produced together with maximal intersection yields a geometry over the diagram of Figure 1 that admits a flag-transitive action of $\mathrm{O}^{\prime} \mathrm{N}$. There is no proof of this assertion in [24]. To the best of our knowledge we provide the first proof of Ivanov-Shpectorov's assertion.

The reason behind our names for types is the following. Points are 0-elements and edges are 1-elements. The cliques of 12-points are 4-elements because the residue of such an element is a geometry of rank 4 with a connected diagram. Residues of the 6-cliques are geometries with a disconnected diagram: the direct sum of an icosahedron and a rank one geometry. It is then intrinsically a geometry of rank 3. The last elements are of type $h$ to remind the word 'hyperline'. As we will detail in Section 10, those elements are endowed with remarkable properties. The name 'hyperline' is an analogy of the French word hyperdroite used by Tits to describe objects in geometries of exceptional type $E_{n}, n=6,7,8$. The first occurence of that terminology can be found in [33, Page 25].

\section{A rank 3 geometry for $\mathrm{O}^{\prime} \mathrm{N}$ due to Connor}

In [15] the second author provides a construction of a rank 3 coset geometry $\Gamma\left(\mathrm{O}^{\prime} \mathrm{N}\right)$ for $\mathrm{O}^{\prime} \mathrm{Nan}$ 's sporadic simple group $\mathrm{O}^{\prime} \mathrm{N}$ over the set of types $I=\{0,1,2\}$. In the present work we denote $\Gamma\left(\mathrm{O}^{\prime} \mathrm{N}\right)$ by $\Gamma_{\mathrm{Co}}$. The geometry $\Gamma_{\mathrm{Co}}$ belongs to the diagram of Figure 2.

Connor starts with coset geometries of rank 2 for $\mathrm{M}_{11}$ and $\mathrm{J}_{1}$ whose dia- 


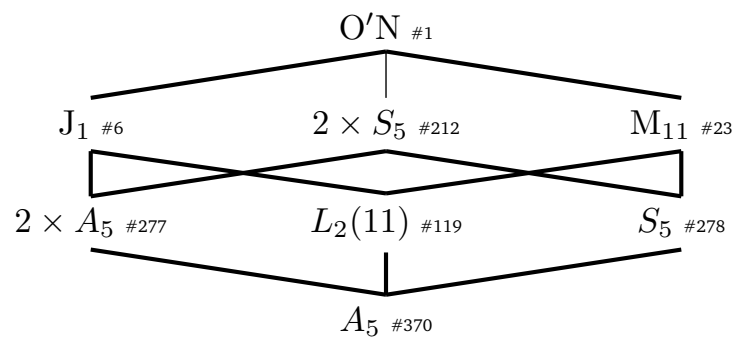

Figure 12: An identification of the boolean lattice of $\Gamma_{\mathrm{Co}}$ in $\Lambda_{\mathrm{O}^{\prime} \mathrm{N}}$

grams are given in Figure 11 [27]. Both geometries have a maximal parabolic subgroup isomorphic to $L_{2}(11)$ and a Borel subgroup isomorphic to $A_{5}$. This observation led him to consider the amalgam of the two diagram geometries in the group $\mathrm{O}^{\prime} \mathrm{N}$ in which $\mathrm{M}_{11}$ and $\mathrm{J}_{1}$ are maximal subgroups. The boolean lattices of the two geometries are given by their Buekenhout diagram. Connor shows that the amalgam of the boolean lattices can be done in a unique way up to conjugacy in $\operatorname{Aut}\left(\mathrm{O}^{\prime} \mathrm{N}\right)$. The resulting boolean lattice of rank 3 is given in Figure 12. Using a theorem due to Aschbacher [1], Connor proves that this boolean lattice yields a firm, residually connected, flag-transitive coset geometry $\Gamma_{\mathrm{Co}}$ over the diagram of Figure 2 . Then he proves that $\Gamma_{\mathrm{Co}}$ is residually weakly primitive, locally 2 -transitive and that it satisfies the intersection property in rank 2 residues. Finally he proves that the automorphism group of the geometry $\Gamma_{\mathrm{Co}}$ is $\mathrm{O}^{\prime} \mathrm{N}$, using a study of the truncation of $\Gamma_{\mathrm{Co}}$ on its elements of type in $\{0,1\}$.

The construction of $\Gamma_{\mathrm{Co}}$ in [15] is independent from [24].

\section{From the Ivanov-Shpectorov geometry to the Con- nor geometry}

So far we have explained the various starting concepts and results as mentionned in the abstract and the introduction. We now turn to new results.

From now on and throughout the remaining of this paper, let $G$ denote a copy of $\mathrm{O}^{\prime} \mathrm{N}$. Let us start with the diagram of the Ivanov-Shpectorov geometry $\Gamma_{\text {IvSh }}=\Gamma\left(G,\left\{G_{0}, G_{1}, G_{3}, G_{4}, G_{h}\right\}\right)$ as in Figure 1. As we observed in Section 5, the parabolic subgroup $G_{0}$ is isomorphic to $\mathrm{J}_{1}$ and the parabolic subgroup $G_{4}$ is isomorphic to $\mathrm{M}_{11}$. The residue $\Gamma_{0}$ of $\Gamma_{\mathrm{IvSh}}$ is the diagram geometry described in Section 4.3 (see also Figure 5) and the residue $\Gamma_{4}$ is the diagram geometry described in Section 4.2 (see also Figure 4). Thanks to Section 4, we know 
that $\mathrm{M}_{11}$ and $\mathrm{J}_{1}$ have unique flag-transitive geometries with those $\Gamma_{\text {IvSh }}$ residue diagrams. Hence we know that the residues $\Gamma_{0}$ and $\Gamma_{4}$ of the geometry $\Gamma_{\text {IvSh }}$ are isomorphic to $\Gamma_{\mathrm{J}_{1}}$ and $\Gamma_{\mathrm{M}_{11}}$ respectively. The observations made in Section 5 show that $G_{1}$ is isomorphic to $2 \times S_{5}$. This leads us to ask whether the truncation of $\Gamma_{\text {IvSh }}$ on the elements of type in $\{0,1,4\}$ could be the Connor geometry $\Gamma_{\mathrm{Co}}$ (see Figure 2). Then the use of the subgroup lattice $\Lambda_{\mathrm{O}^{\prime} \mathrm{N}}$ of $\mathrm{O}^{\prime} \mathrm{N}$ readily shows that these data provide indeed the needed diagram geometry as is shown in Theorem 7.1. This provides an alternative construction of the Connor geometry from the knowledge provided in [24].

Theorem 7.1. The Connor geometry $\Gamma_{\mathrm{Co}}$ is a truncation of the Ivanov-Shpectorov geometry $\Gamma_{\mathrm{IvSh}}$ on the set of types $\{0,1,4\}$.

Proof. The strategy of the proof is to show that the truncation of $\Gamma_{\text {IvSh }}$ on its elements of type in $\{0,1,4\}$ is a coset geometry with the same boolean lattice as $\Gamma_{\mathrm{Co}}$. Then we make use of results in [15] stating that such a boolean lattice yields a unique flag-transitive geometry with automorphism group isomorphic to $\mathrm{O}^{\prime} \mathrm{N}$.

By the developments of Section 5, we know that $G_{0} \cong \mathrm{J}_{1}, G_{1} \cong 2 \times S_{5}, G_{4} \cong$ $\mathrm{M}_{11}, G_{04} \cong L_{2}(11)$ and $G_{01} \cong 2 \times A_{5}$. Consider a flag $F$ of types in $\{0,1,4\}$. By Assertion 1 in [24], the residue of $F$ is isomorphic to the Petersen graph and it is flag-transitive. Since it has $10 \times 3=30$ flags, the group $G_{014}$ must have order divisible by 30 . Moreover this group must be isomorphic to a subgroup of $2 \times S_{5}$, $L_{2}(11)$ and $\mathrm{M}_{11}$ (see Tables 6, 7 and 4). By looking at the subgroup lattices of each of these groups, it is easily seen that $G_{014} \cong A_{5}$. Moreover $G_{14}$ must strictly contain a subgroup isomorphic to $A_{5}$ and must be strictly contained in a subgroup isomorphic to $2 \times S_{5}$. Consequently, $G_{14} \cong 2 \times A_{5}$ or $G_{14} \cong S_{5}$. Since $\mathrm{M}_{11}$ does not have a subgroup isomorphic to $2 \times A_{5}$, we get $G_{14} \cong S_{5}$. We just showed that the boolean lattice of Figure 12 is a rank 3 sublattice of the boolean lattice of $\Gamma_{\text {IvSh. }}$. By Theorem 4.1 of [15], there are two such boolean lattices of rank 3 up to conjugacy in $\mathrm{O}^{\prime} \mathrm{N}$ and by Theorem 4.2 in [15] each of them determines a geometry isomorphic to $\Gamma_{\mathrm{Co}}$. Those two families of rank 3 boolean lattices are fused in $\operatorname{Aut}\left(\mathrm{O}^{\prime} \mathrm{N}\right) \cong \mathrm{O}^{\prime} \mathrm{N}: 2$.

Theorem 7.1 is the first result announced in our abstract.

\section{Identification of $L_{\mathrm{IvSh}}$ in $\Lambda_{\mathrm{O}^{\prime} \mathrm{N}}$}

The group $G \cong \mathrm{O}^{\prime} \mathrm{N}$ acts flag-transitively on the geometry $\Gamma_{\mathrm{IvSh}}$. Using the Tits construction described in Section 2.3, we can see $\Gamma_{\mathrm{IvSh}}$ as a coset geometry 


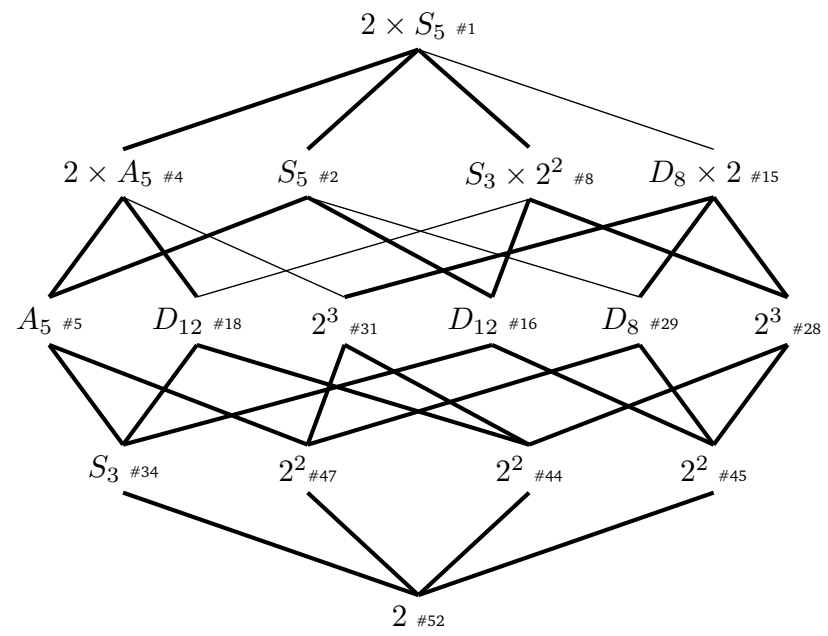

Figure 13: The boolean lattice of $\Gamma_{2 \times S_{5}}$ identified in $\Lambda_{2 \times S_{5}}$

$\Gamma\left(G,\left\{G_{0}, G_{1}, G_{3}, G_{4}, G_{h}\right\}\right)$. In this section we determine the boolean lattice $L_{\mathrm{IvSh}}$ of parabolic subgroups of this coset geometry on the basis of Section 5 .

As a matter of fact, we know four maximal parabolic subgroups: $G_{0} \cong \mathrm{J}_{1}$, $G_{1} \cong 2 \times S_{5}, G_{4} \cong \mathrm{M}_{11}$, and $G_{3} \cong A_{5} \times S_{3}$ (see Section 5). Moreover we proved in Section 4 that $\mathrm{J}_{1}$ and $\mathrm{M}_{11}$ have unique flag-transitive geometries over the residue diagrams provided by the diagram of $\Gamma_{\text {IvSh }}$ and we computed the boolean lattice of each of them in Section 4.4.

The residue $\Gamma_{1}$ (resp. $\Gamma_{3}$ ) is a geometry with $2 \times S_{5}$ (resp. $A_{5} \times S_{3}$ ) acting flag-transitively. We denote this geometry by $\Gamma_{2 \times S_{5}}$ (resp. $\Gamma_{A_{5} \times S_{3}}$ ) to emphasize the group action.

We now further analyze the residue $\Gamma_{1} \cong \Gamma_{2 \times S_{5}}$. The stabilizer of a point in the residue $\Gamma_{0} \cong \Gamma_{J_{1}}$ is also a maximal parabolic subgroup of $\Gamma_{1} \cong \Gamma_{2 \times S_{5}}$. Hence a maximal parabolic subgroup of $\Gamma_{2 \times S_{5}}$ is isomorphic to $2 \times A_{5}$. This subgroup acts on the direct sum of the rank 2 geometry of a Petersen graph and a rank 1 geometry of 2 points. A parabolic subgroup isomorphic to $A_{5}$ acts flagtransitively on the Petersen graph. Table 6 shows that this subgroup belongs to conjugacy class $\# 5$ in $\Lambda_{2 \times S_{5}}$. Moreover a subgroup of class $\# 5$ has exactly three overgroups in $2 \times S_{5}$ : an overgroup isomorphic to $2 \times A_{5}$ belonging to class \#4 and two overgroups isomorphic to $S_{5}$ belonging to classes \#3 and \#2 respectively. Consequently a maximal parabolic subgroup of $\Gamma_{2 \times S_{5}}$ is isomorphic to $S_{5}$ and acts on a direct sum of a Petersen graph and a rank 1 geometry of 2 points. This coset geometry of rank 3 is already known (see for instance [11, page 36]). At this point, knowing already two maximal parabolic subgroups, it is an easy 


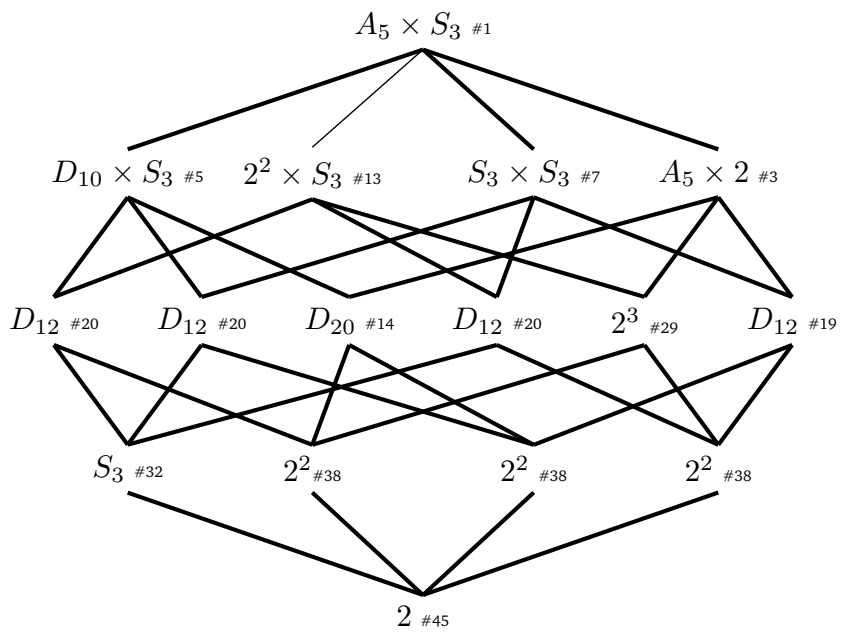

Figure 14: The boolean lattice of $\Gamma_{A_{5} \times S_{3}}$ identified in $\Lambda_{A_{5} \times S_{3}}$

task to compute the whole boolean lattice $L_{2 \times S_{5}}$ of $\Gamma_{2 \times S_{5}}$ as in Figure 13 .

Let us mention further that geometries for $2 \times S_{5}$ have been studied by Cara and Leemans [14]. They classified the residually weakly primitive geometries for this group and so they knew geometry $\Gamma_{1}$.

Let us now focus on $\Gamma_{3} \cong \Gamma_{A_{5} \times S_{3}}$. In this case too we know already some parabolic subgroups thanks to our conclusions on the residues $\Gamma_{0} \cong \Gamma_{J_{1}}, \Gamma_{1} \cong$ $\Gamma_{2 \times S_{5}}$ and $\Gamma_{4} \cong \Gamma_{\mathrm{M}_{11}}$. We leave the reader checking for himself that the boolean lattice $L_{A_{5} \times S_{3}}$ of the coset geometry $\Gamma_{3}$ is as depicted in Figure 14 by making use of the subgroup lattice $\Lambda_{A_{5} \times S_{3}}$ given in Table 8 .

The boolean lattices $L_{\mathrm{J}_{1}}, L_{\mathrm{M}_{11}}, L_{2 \times S_{5}}$ and $L_{A_{5} \times S_{3}}$ of the four coset geometries $\Gamma_{\mathrm{J}_{1}}, \Gamma_{2 \times S_{5}}, \Gamma_{\mathrm{M}_{11}}$ and $\Gamma_{A_{5} \times S_{3}}$ occur as sublattices in the boolean lattice $L_{\mathrm{IvSh}}$ of $\Gamma_{\mathrm{IvSh}}$. Our strategy to compute $L_{\mathrm{IvSh}}$ is to use that knowledge by filling in a general rank 5 boolean lattice with 32 unknowns with those four rank 4 boolean lattices (see Figures 15 and 16). We prove that this can be done in a unique way up to conjugacy in $\operatorname{Aut}\left(\mathrm{O}^{\prime} \mathrm{N}\right)$. There remains an unknown in this boolean lattice, namely $G_{h}$, and we prove that it is in fact uniquely determined in Theorem 8.1 under the assumption that the resulting boolean lattice is generating (see proof of Lemma 3.1). This will finish the computation of the boolean lattice of $\Gamma_{\text {IvSh }}$, the second result stated in our abstract.

Afterwards we start with the boolean lattice of subgroups of $L_{\mathrm{IvSh}}$ identified in the subgroup lattice of $\mathrm{O}^{\prime} \mathrm{N}$ and we prove in Theorem 9.4 that it yields a flag-transitive, residually connected, firm geometry. This is a new construction 


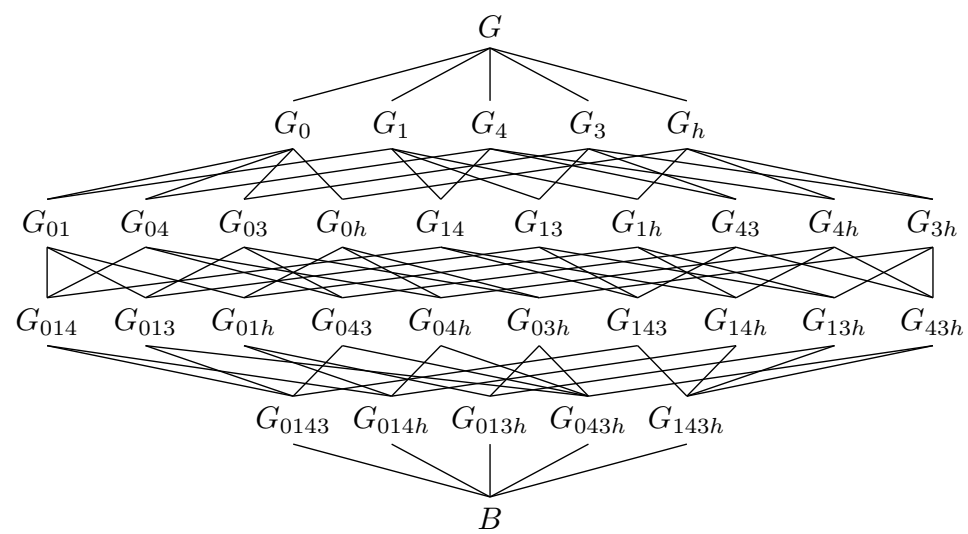

Figure 15: The general boolean lattice of a rank 5 coset geometry with set of types according to our choice for $\Gamma_{\mathrm{IvSh}}$

of $\Gamma_{\text {IvSh }}$ from the subgroup lattice of $\mathrm{O}^{\prime} \mathrm{N}$.

\subsection{Existence and uniqueness of $\Gamma_{\text {IvSh }}$ 's boolean lattice in $\mathrm{O}^{\prime} \mathrm{N}$}

We prove the following theorem on the basis of the developments of Section 5 .

Theorem 8.1. Up to conjugacy, there are exactly two partial boolean lattices as in Figure 16 in the subgroup lattice of $\mathrm{O}^{\prime} \mathrm{N}$. Each of them extends uniquely to a generating boolean lattice of subgroups of $\mathrm{O}^{\prime} \mathrm{N}$ as in Figure 17. Those two families of lattices are fused in $\operatorname{Aut}\left(\mathrm{O}^{\prime} \mathrm{N}\right) \cong \mathrm{O}^{\prime} \mathrm{N}: 2$.

\subsection{Proof of Theorem 8.1}

Table 9 contains carefully chosen subgroups of $\mathrm{O}^{\prime} \mathrm{N}$ extracted from $\Lambda_{\mathrm{O}^{\prime} \mathrm{N}}$ in order to help the reader during the process of the upcoming proof. Labels that matter in that proof are bold to ease the reading.

There is exactly one conjugacy class of subgroups isomorphic to $J_{1}$ in $\mathrm{O}^{\prime} \mathrm{N}$. Thanks to Theorem 4.4 it is quite straightforward to identify the boolean lattice of $\Gamma_{J_{1}}$ in $\Lambda_{\mathrm{O}^{\prime} \mathrm{N}}$ depicted in Figure 18. Since our aim is now to extend this boolean lattice of rank 4 to a boolean lattice of rank 5 , it is convenient to provide parabolic subgroups of $\Gamma_{J_{1}}$ embedded in $\mathrm{O}^{\prime} \mathrm{N}$ with names suited to our purpose. This is done by the natural comparison between Figure 15 and Figure 16. Namely $G_{0} \cong \mathrm{J}_{1}, G_{01} \cong 2 \times A_{5}, G_{04} \cong L_{2}(11), G_{03} \cong S_{3} \times D_{10}$, 


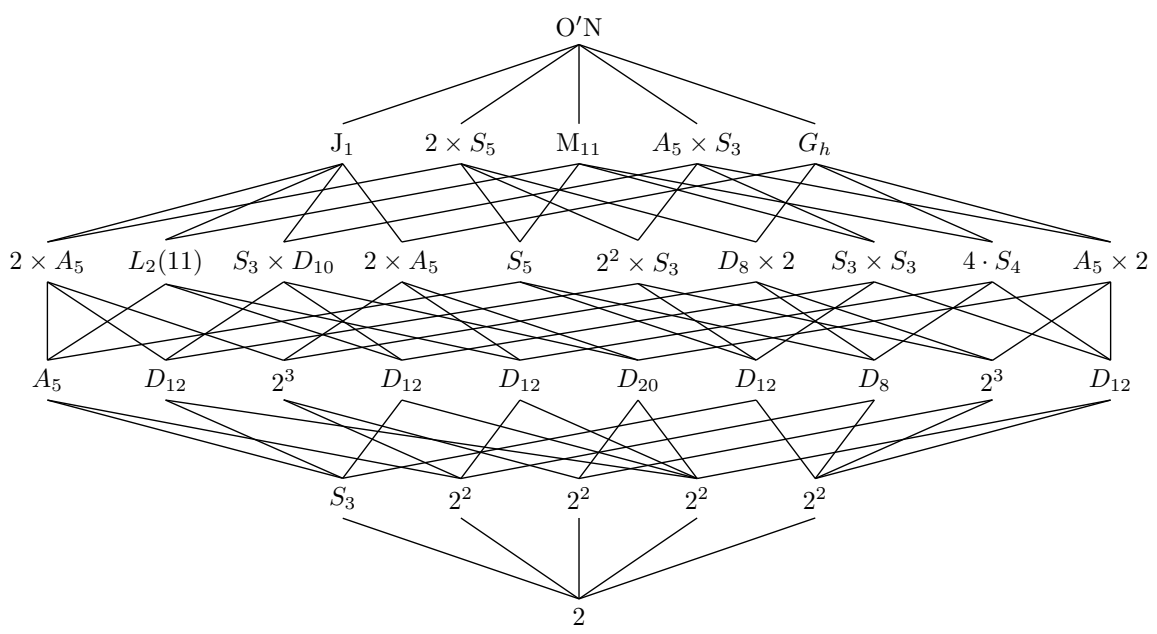

Figure 16: The boolean lattices of $\Gamma_{\mathrm{J}_{1}}, \Gamma_{2 \times S_{5}}, \Gamma_{\mathrm{M}_{11}}$ and $\Gamma_{A_{5} \times S_{3}}$ amalgamated in a rank 5 boolean lattice with a unique unknown

$G_{0 h} \cong 2 \times A_{5}, G_{014} \cong A_{5}, G_{013} \cong D_{12}, G_{01 h} \cong 2^{3}, G_{043} \cong D_{12}, G_{04 h} \cong D_{12}$, $G_{03 h} \cong D_{20}, G_{0143} \cong S_{3}, G_{014 h} \cong G_{013 h} \cong G_{043 h} \cong 2^{2}$ and $B \cong 2$.

Notice that a subgroup isomorphic to $L_{2}(11)$ (necessarily in class \#119) is a maximal subgroup of two subgroups of $\mathrm{O}^{\prime} \mathrm{N}$ isomorphic to $\mathrm{M}_{11}$ (in classes $\# 23$ and \#24) and one subgroup isomorphic to $\mathrm{J}_{1}$. Hence we can choose the subgroups $G_{0} \cong \mathrm{J}_{1}$ and $G_{4} \cong \mathrm{M}_{11}$ in $\mathrm{O}^{\prime} \mathrm{N}$ in classes \#6 and \#23 respectively such that $G_{04}=G_{0} \cap G_{4}$ is a subgroup isomorphic to $L_{2}(11)$ of class \#119. The parabolic subgroup $G_{014} \cong A_{5}$ belongs to class \#370. By construction, $G_{014} \cong$ $A_{5}$ (in class \#370) is a subgroup of $G_{4} \cong \mathrm{M}_{11}$. Table 9 shows that a subgroup of class \#370 sits in exactly two overgroups of class \#278, isomorphic to $S_{5}$. On the other hand $\mathrm{M}_{11}$ has a unique class of (maximal) subgroups isomorphic to $S_{5}$. In the case of $G_{4}$, that class of $S_{5}$ is \#278. Therefore we can choose a subgroup $G_{14} \cong S_{5}$ in class \#278 contained in $G_{4}$ and containing $G_{014}$. Now $\left\langle G_{01}, G_{14}\right\rangle$ is by definition the smallest common overgroup of $G_{01}$ and $G_{14}$. In this situation, it should be clear that the group they generate is a subgroup of $\mathrm{O}^{\prime} \mathrm{N}$ of class \#212, isomorphic to $2 \times S_{5}$ (compare with Table 6). Observe at this point that we just proved Theorem 4 stated in [15].

By Theorem 4.3 we may choose subgroups of $\mathrm{M}_{11} \leq \mathrm{O}^{\prime} \mathrm{N}$ such as in Figure 9 . This provides us with $G_{43} \cong S_{3} \times S_{3}$ in class \#407, $G_{4 h} \cong 2 \cdot S_{4}$ in class $\# 382, G_{143} \cong D_{12}$ in class \#537, $G_{14 h} \cong D_{8}$ in class \#564 and $G_{143 h} \cong 2^{2}$ in class \#578. At this stage, we have already extracted 25 subgroups of $\mathrm{O}^{\prime} \mathrm{N}$ that intersect pairwise in such a way that they form a partial boolean lattice. The 

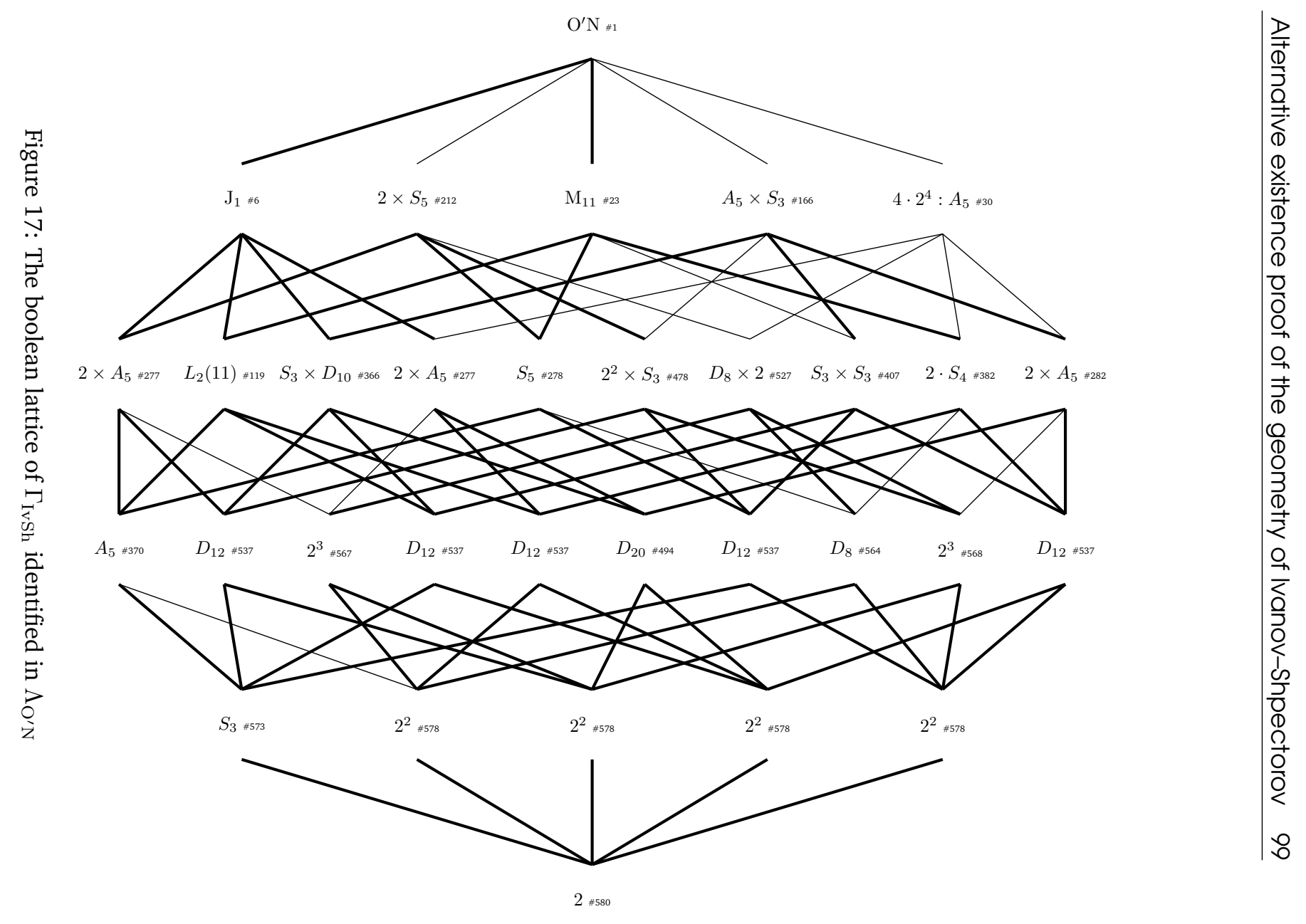
seven remaining unknowns are $G_{3}, G_{h}, G_{13}, G_{1 h}, G_{3 h}, G_{13 h}$ and $G_{14 h}$.

In Section 8 we extracted a boolean lattice of subgroups $\Gamma_{2 \times S_{5}}$ from the subgroup lattice of $2 \times S_{5}$. We apply this construction here to $G_{1} \cong 2 \times S_{5}$ and we obtain $G_{13} \cong 2^{2} \times S_{3}$ in class \#478, $G_{1 h} \cong Q_{8}: 2$ in class \#527, $G_{13 h} \cong 2^{3}$ in class \#568 and $G_{14 h} \cong D_{12}$ in class \#537. This leaves us with three unknowns namely $G_{3}, G_{h}$ and $G_{3 h}$.

$G_{03} \cong S_{3} \times D_{10}$ has exactly one overgroup isomorphic to $A_{5} \times S_{3}$ in class \#166. A subgroup of $\mathrm{O}^{\prime} \mathrm{N}$ of class \#166 contains subgroups of class \#407. Therefore we can assume that the overgroup of $G_{03}$ contains $G_{43} \cong S_{3} \times S_{3}$ in class \#407, for otherwise we choose conjugate subgroups to $G_{43}$ in $G_{4}$. Similarly, we can assume that it contains $G_{13} \cong 2^{2} \times S_{3}$ in class \#478. Hence we can assume that subgroups $G_{03}, G_{13}$ and $G_{43}$ generate $G_{3} \cong A_{5} \times S_{3}$ in class \#166. Now we apply the construction of $\Gamma_{A_{5} \times S_{3}}$ given in Section 8 which allows us to choose $G_{3 h}$ as a subgroup isomorphic to $2 \times A_{5}$ which must belong to class \#282.

For the last unknown $G_{h}$, careful observation of the respective overgroups of $G_{0 h}, G_{1 h}, G_{4 h}, G_{3 h}$ shows that their least possible common overgroup in $\mathrm{O}^{\prime} \mathrm{N}$ is a subgroup of class \#30 isomorphic to $4 \cdot 2^{4}: A_{5}$ or one of its overgroups. However a subgroup of class \#30 is quasimaximal, i.e. it has a unique chain of overgroups with maximal element $\mathrm{O}^{\prime} \mathrm{N}$. Hence the least common overgroup of $G_{0 h}, G_{1 h}, G_{4 h}, G_{3 h}$ is either a subgroup of class \#30 or $G$ itself. If it were $G$, then $\Gamma_{\text {IvSh }}$ would be degenerate.

The configuration of 32 subgroups of $\mathrm{O}^{\prime} \mathrm{N}$ as we just produced yields a boolean lattice of subgroups. Observe moreover that it is generating by construction.

\section{Construction of the geometry $\Gamma_{\operatorname{IvSh}}$}

We prove that the $\Gamma_{\text {IvSh }}$ boolean lattice described in Section 8 is the boolean lattice of a unique firm, residually connected, flag-transitive geometry for $\mathrm{O}^{\prime} \mathrm{N}$. Then we prove that this geometry is the geometry $\Gamma_{\mathrm{IvSh}}$ built in [24].

\subsection{Graphs for $\mathrm{O}^{\prime} \mathrm{N}$ and $\mathrm{O}^{\prime} \mathrm{N}: 2$}

The truncation of $\Gamma_{\text {IvSh }}$ on the set of types $\{0,1\}$ is a graph $\mathcal{G}_{\text {IvSh }}$ of which objects of type 3,4 and $h$ are subgraphs. We construct and describe this graph in this section. We first define the $2 \mathrm{~B}$-commuting involution graph of $\mathrm{O}^{\prime} \mathrm{N}: 2$. Then we describe some of the action of $\mathrm{O}^{\prime} \mathrm{N}<\mathrm{O}^{\prime} \mathrm{N}: 2$ on that graph in order to produce the graph $\mathcal{G}_{\mathrm{IvSh}}$. 


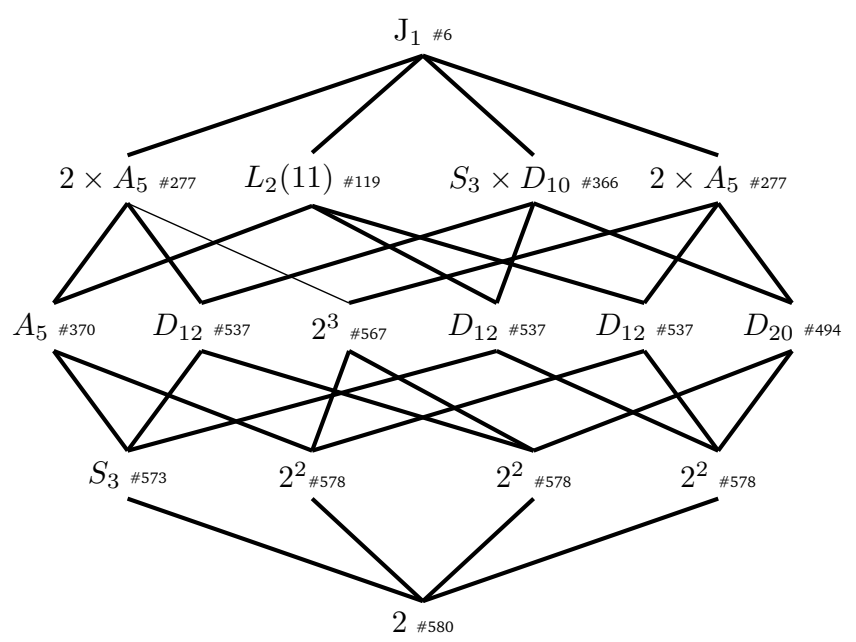

Figure 18: The boolean lattice of $\Gamma_{J_{1}}$ identified in $\Lambda_{\mathrm{O}^{\prime} \mathrm{N}}$

\subsubsection{The commuting 2B-involution graph of $\mathrm{O}^{\prime} \mathrm{N}: 2$}

The group $\operatorname{Aut}(G) \cong \mathrm{O}^{\prime} \mathrm{N}: 2$ has two classes of involutions namely $2 \mathrm{~A}$ and $2 \mathrm{~B}$ according to the notation of the ATLAS [17]. Let $\rho$ be a 2B-involution (an outer automorphism of $G$ ). The centralizer $C:=\mathrm{C}_{\operatorname{Aut}(G)}(\rho)$ of $\rho$ in $\operatorname{Aut}(G)$ is isomorphic to $\mathrm{J}_{1} \times 2$. We define the graph $\mathcal{C}=\left(V_{\mathcal{C}}, E_{\mathcal{C}}\right)$ as follows. The set $V_{\mathcal{C}}$ of vertices of $\mathcal{C}$ is the set of $2 \mathrm{~B}$-involutions of $\mathrm{O}^{\prime} \mathrm{N}: 2$. The set $E_{\mathcal{C}}$ of edges of $\mathcal{C}$ consists of the pairs of $2 \mathrm{~B}$-involutions that commute. Such a graph is called a commuting involution graph [2]. The graph $\mathcal{C}$ has 2624832 vertices and 1920064608 edges. It is arc-transitive of valency 1463.

Lemma 9.1. The centralizer $C$ has an orbit $\Sigma$ of size 2926 on the set of vertices of $\mathcal{C}$.

Proof. We work in the subgroup lattice $\Lambda_{2}$ of $\operatorname{Aut}(G) \cong \mathrm{O}^{\prime} \mathrm{N}: 2$ and we consider the conjugacy class of 2B-involutions of $\operatorname{Aut}(G)$ that correspond to class \#732 in $\Lambda_{2}$. 


\begin{tabular}{|c|c|c|c|c|c|}
\hline Nr. & Structure & Order & Length & Maximal Subgroups & Minimal Overgroups \\
\hline 712 & $D_{8}$ & 8 & 960032304 & 727 (2), 728 & $\begin{array}{l}430(6), 493(20), 531 \\
(6), 532(30), 620(10), \\
625(10), 656(10), 662 \\
(15)\end{array}$ \\
\hline 713 & $D_{8}$ & 8 & 14400484560 & 727 (2), 726 & $\begin{array}{l}431(2), 440(4), 533 \\
(4), 626(4), 654 \text { (2), } \\
662,666(2)\end{array}$ \\
\hline 716 & $2^{3}$ & 4 & 4800161520 & $730,727(6)$ & $\begin{array}{l}529(12), 610(12), 622 \\
(4), 662(3), 666 \text { (3), } \\
667\end{array}$ \\
\hline 727 & $2^{2}$ & 4 & 1920064608 & $733,732(2)$ & $\begin{array}{l}412(40), 520(24), 605 \\
(20), 606(40), 633 \\
(60), 635(20), 638 \\
(6), 639(30), 685(60), \\
686(10), 687(12), 692 \\
(10), 712,713(15), \\
716(15)\end{array}$ \\
\hline 732 & 2 & 2 & 2624832 & 734 & $\begin{array}{lrr}479(11704), & 535 \\
(29260), \quad 536 & (1540), \\
628 \quad(1596), & 629 \\
(17556), 676 & (4180), \\
680(29260), & 681 \\
(4180), 696 & (2926), \\
699(14630), & 721 \\
(2926), 724 & (2926), \\
727(1463) & \end{array}$ \\
\hline
\end{tabular}

Table 2: Overgroups of order 8 of a $2 \mathrm{~B}$-involution in $\mathrm{O}^{\prime} \mathrm{N}: 2$

Table 2 is extracted from $\Lambda_{2}$ and shows sublattices of subgroups of $\operatorname{Aut}(G)$ of order 8 that contain a 2B-involution. Two of them are dihedral groups of order 8 , namely subgroups of classes \#712 and \#713. The dihedral group $D_{8}$ contains 5 involutions. Seeing $D_{8}$ as the automorphism group of a square $S$, we can give a geometric interpretation of those 5 involutions: they are the central symmetry of $S$, two symmetries around axes through opposite vertices of $S$ and two symmetries around axes through midpoints of opposite edges of $S$. Considering Table 2, we see that dihedral subgroups of class \#712 have four 2Binvolutions and one (central) $2 \mathrm{~A}$-involution. Out of the four 2B-involutions, one is $\rho$ and another one is an involution $\rho^{\prime}$ that commutes with $\rho$. The involution $\rho^{\prime}$ belongs to the orbit of size 1463 of $\mathcal{C}$ under the action of $C$ since $\rho^{\prime}$ is a neighbor of $\rho$ in $\mathcal{C}$. The remaining two 2B-involutions $\sigma$ and $\sigma^{\prime}$ commute together but do not commute with either $\rho$ or $\rho^{\prime}$. Moreover we read from Table 2 that $\rho$ belongs to 1463 dihedral subgroups of class \#712 which yield 1463 2B-involutions that commute with $\rho$. Thus any dihedral subgroup of class \#712 also yields two more 2B-involutions. There are $2 \times 1463=29262 \mathrm{~B}$-involutions arising in this way. 
Let us call $\Sigma$ the set of those 2926 2B-involutions. Since $C$ is transitive on the involutions commuting with $\rho$ and since a pair $\left(\rho, \rho^{\prime}\right)$ of commuting involutions yields a dihedral subgroup of class \#712, we conclude that $\Sigma$ is an orbit under the action of $C=\operatorname{Stab}_{\operatorname{Aut}(G)}(\rho)=C_{\operatorname{Aut}(G)}(\rho)$.

We determine with MAGMA that the full orbit distribution of $C$ on 2624832 points is

$1+1463+2926+5852+25080+29260^{3}+43890+58520^{3}+87780^{2}+175560^{10}+351120$.

\subsubsection{The Ivanov-Shpectorov graph for $\mathrm{O}^{\prime} \mathrm{N}$}

Lemma 9.2. The index 2 subgroup $J \cong \mathrm{J}_{1}$ of $C$ has two orbits on the set $\Sigma$, each of size 1463 .

Proof. The stabilizer in $J$ of a point of $\Sigma$ is isomorphic to $2 \times A_{5}$ (the centralizer of an involution in $\mathrm{J}_{1}$ ), which is a maximal subgroup of index 1463 of $J$. Therefore $J$ acts intransitively on $\Sigma$ with two orbits of size 1463 each.

Consider the action of $J<C=C_{\mathrm{Aut}_{\left(\mathrm{O}^{\prime} \mathrm{N}\right)}}(\rho)$ on the set of vertices of $\mathcal{C}$. It is now clear that $J$ has three orbits $O_{1463}^{1}, O_{1463}^{2}, O_{1463}^{3}$ of size 1463 , namely the orbit $O_{1463}^{1}$ of 2B-involutions commuting with $\rho$ and two orbits $O_{1463}^{2}$ and $O_{1463}^{3}$, each of size 1463 such that $O_{1463}^{2} \sqcup O_{1463}^{3}=\Sigma$. Let $\alpha \in O_{1463}^{1}, \beta \in O_{1463}^{2}$ and $\gamma \in O_{1463}^{3}$.

\begin{tabular}{||c|l|c|c|l|l||}
\hline Nr. & Structure & Order & Length & Maximal Subgroups & Minimal Overgroups \\
\hline 1 & $\mathrm{O}^{\prime} \mathrm{N}$ & 460815505920 & 1 & $2(122760)$, & \\
& & & & $3(122760)$, & \\
& & & & $6(2624832)$, & \\
& & & $7(2857239)$, & \\
& & & $11(17778376)$, & \\
& & & $12(17778376)$, & \\
& & & $15(30968784)$, & \\
& & & $16(30968784)$, & \\
& & & $21(42858585)$, & \\
& & & $23(58183776)$, & \\
& & & $24(58183776)$, & \\
& & & $43(182863296)$, & \\
\hline
\end{tabular}




\begin{tabular}{|c|c|c|c|c|c|}
\hline \multicolumn{6}{|c|}{ continued from previous page } \\
\hline Nr. & Structure & Order & Length & Maximal Subgroups & Minimal Overgroups \\
\hline 6 & $\mathrm{~J}_{1}$ & 175560 & 2624832 & $\begin{array}{l}119(266), \\
237(1045), \\
277(1463), \\
285(1540), \\
286(1596), \\
366(2926), \\
397(4180)\end{array}$ & 1 \\
\hline 7 & $4 \cdot L_{3}(4): 2$ & 161280 & 2857239 & 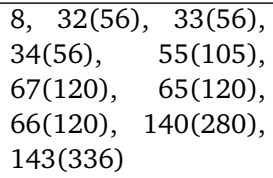 & 1 \\
\hline 8 & $4 \cdot L_{3}(4)$ & 80640 & 2857239 & $\begin{array}{lr}30(42), & 57(56), \\
58(56), & 59(56), \\
112(120), & 113(120), \\
114(120), & 194(280)\end{array}$ & 7 \\
\hline 30 & $4 \cdot 2^{4}: A_{5}$ & 3840 & 120004038 & $\begin{array}{lr}97(5), & 133(6), \\
156(10), & 208(16), \\
209(16), & 210(16), \\
211(16) & \end{array}$ & 8 \\
\hline 143 & $A_{5}: D_{8}$ & 480 & 960032304 & $\begin{array}{l}212, \quad 213, \quad 210, \\
299(5), \\
386(10)\end{array}$ & 7 \\
\hline 210 & $4 \times A_{5}$ & 240 & 960032304 & $\begin{array}{l}277,391(5), 403(6), \\
481(10)\end{array}$ & $30(2), 143$ \\
\hline 212 & $2 \times S_{5}$ & 240 & 960032304 & $\begin{array}{l}278(2), 277,393(5), \\
402(6), 478(10)\end{array}$ & 143 \\
\hline 213 & $2 \times S_{5}$ & 240 & 960032304 & $\begin{array}{l}279(2), 277,394(5), \\
401(6), 479(10)\end{array}$ & 143 \\
\hline 277 & $2 \times A_{5}$ & 120 & 960032304 & $\begin{array}{l}370,482(5), 494(6), \\
537(10)\end{array}$ & $6(4), 210,212,213$ \\
\hline
\end{tabular}

Table 3: Overgroups of a subgroup of class \#277 in $\Lambda_{\mathrm{O}^{\prime} \mathrm{N}}$

Lemma 9.3. We have $\operatorname{Stab}_{G}\{\rho, \alpha\} \cong 4 \times A_{5}, \operatorname{Stab}_{G}\{\rho, \beta\} \cong \operatorname{Stab}_{G}\{\rho, \gamma\} \cong$ $2 \times S_{5}$. Moreover $\operatorname{Stab}_{G}\{\rho, \beta\}$ and $\operatorname{Stab}_{G}\{\rho, \gamma\}$ are not conjugate in $G$.

Proof. Consider a subgroup $2 \times A_{5} \cong A<J$. It belongs to class $\# 277$ in $\Lambda_{\mathrm{O}^{\prime} \mathrm{N}}$. The lattice of overgroups of $A$ extracted from $\Lambda_{\mathrm{O}^{\prime} \mathrm{N}}$ is pictured in Table 3 . We see that $A$ is in exactly four distinct subgroups of $G$ isomorphic to $\mathrm{J}_{1}$. Therefore $A$ fixes four points of $\mathcal{C}$. The normalizer $N:=\mathrm{N}_{G}(A) \cong A_{5}: D_{8} \cong\left(A_{5} \times 2\right) \cdot 2^{2}$ stabilizes those four points and acts transitively on them. We see in Table 3 that there are exactly three subgroups between $N$ and $A$. They are subgroups of classes \#212, \#213 and \#210. Since $N$ acts transitively on 4 points with kernel $2 \times A_{5}$, each of its subgroups $2 \times S_{5}^{A}$ and $2 \times S_{5}^{B}$ fixes two points and exchanges the remaining two while $4 \times A_{5}$ has no fixed point. Moreover $\operatorname{Aut}(G)$ fuses the 


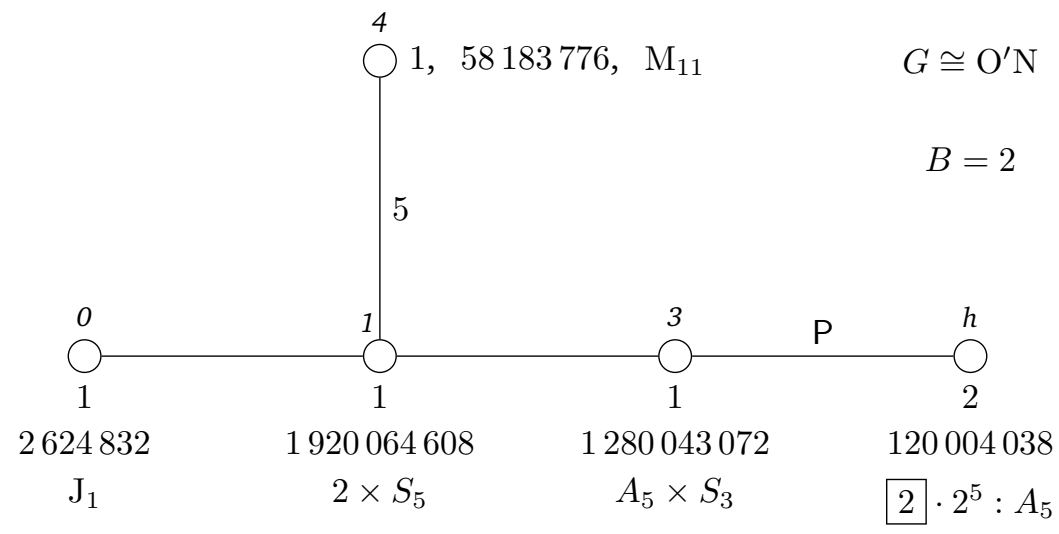

Figure 19: The Buekenhout diagram of $\Gamma_{\text {IvSh }}$

two conjugacy classes \#212 and \#213. Therefore we have $\operatorname{Stab}_{G}\{\rho, \alpha\} \cong 4 \times A_{5}$, $\operatorname{Stab}_{G}\{\rho, \beta\} \cong 2 \times S_{5}^{A}$ and $\operatorname{Stab}_{G}\{\rho, \gamma\} \cong 2 \times S_{5}^{B}$.

We are now able to define a graph $\mathcal{G}_{\mathrm{IVSh}}=\left(V_{\mathrm{IvSh}}, E_{\mathrm{IvSh}}\right)$ as follows. The vertex set of $\mathcal{G}_{\text {IvSh }}$ is the set of vertices of $\mathcal{C}$. The set of edges $E_{\mathrm{IvSh}}$ is the set of all the pairs of vertices whose stabilizer in $\mathrm{O}^{\prime} \mathrm{N}$ is a subgroup of class \#212. Definition of $\mathcal{G}_{\text {IvSh }}$ corresponds obviously to the construction of the graph defined in [24]. With MAGMA we compute the distance distribution map of $\mathcal{G}_{\text {IvSh }}$ given in Tables 10, 11 and 12.

\subsection{Existence and uniqueness of a geometry from $L_{\mathrm{IvSh}}$}

We prove the following theorem.

Theorem 9.4. The boolean lattice of Figure 17 is the lattice of parabolic subgroups of a unique firm, residually connected, flag-transitive coset geometry over the Buekenhout diagram in Figure 19.

\subsection{Proof of Theorem 9.4}

First we define a set of types $I=\{0,1,3,4, h\}$ as suggested by Figure 1 . Next we provide the construction of a geometry $\Gamma=(X, *, \tau)$ over the set of types $I$. We define the elements of each type as subsets of vertices of $\mathcal{G}_{\text {IvSh }}$ stabilized by parabolic subgroups of the boolean lattice $L_{\mathrm{IvSh}}$. Then we provide an incidence 
relation and we prove that we obtain a firm, residually connected, flag-transitive geometry.

\subsubsection{Elements of types $0,1,3$ and 4}

Elements of type 0 are the left cosets of $G_{0} \cong \mathrm{J}_{1}$ in $G \cong \mathrm{O}^{\prime} \mathrm{N}$. They are the vertices of the graph $\mathcal{G}_{\text {IvSh }}$ defined in Section 9.1.2. We also call elements of type 0 points. The stabilizer of any point is isomorphic to $\mathrm{J}_{1}$ by construction. Call $p$ the vertex stabilized by $G_{0}$ in $\mathcal{G}_{\mathrm{IvSh}}$. The group $G$ acts primitively on the set $X_{0}$ of points by left translation. (Notice it is also the case for $\mathrm{O}^{\prime} \mathrm{N}: 2 \cong \operatorname{Aut}\left(\mathrm{O}^{\prime} \mathrm{N}\right)$.)

The elements of type 1 are edges of $\mathcal{G}_{\mathrm{IvSh}}$. By Lemma 9.3 the stabilizer of an edge in $\mathcal{G}_{\text {IvSh }}$ is isomorphic to $2 \times S_{5}$ and conjugate to $G_{1}$.

The boolean lattice of subgroups of $G_{4} \cong \mathrm{M}_{11}$ yields the flag-transitive geometry $\Gamma_{\mathrm{M}_{11}}$ constructed in Section 4.2 which has 12 points. Since $\mathrm{M}_{11}$ acts 3 -transitively on those 12 points, $G_{4}$ yields a clique of 12 vertices in $\mathcal{G}_{\mathrm{IvSh}}$. Each left coset of $G_{4}$ in $G$ provides such a 12-clique. They are the 4-elements of $\Gamma$.

The boolean lattice of subgroups of $G_{3} \cong A_{5} \times S_{3}$ yields the flag-transitive geometry $\Gamma_{A_{5} \times S_{3}}$ constructed in Section 8 which has 6 points forming a clique. Each left coset of $G_{3}$ in $G$ provides such a 6 -clique. They are the 3-elements of $\Gamma$.

\subsection{2 $h$-Elements}

By Lemma 9.3 the maximal parabolic subgroup $G_{0} \cong \mathrm{J}_{1}$ acts on three orbits of size 1463 in the same way it acts on its set of involutions. Let $p$ be the point fixed by $G_{0}$. The subgroup $G_{01} \cong 2 \times A_{5}$ is the pointwise stabilizer $\operatorname{Stab}_{G}[p, x]$ of a pair $\{p, x\}, x \in O_{1463}^{2}$. Obviously $\operatorname{Stab}_{G}\{p, x\}$ is conjugate to $G_{1}$ in $G$. Moreover $\operatorname{Stab}_{G}[p, x]$ has two orbits of size 15 in $O_{1463}^{2}$ on which it acts in the same way it acts on its 2-Sylow subgroups isomorphic to $2^{3}$. In particular, $G_{0 h} \cong 2 \times A_{5}$ fixes a point $q$ in $O_{1463}^{2}$ and has two such orbits $O_{15}^{1}$ and $O_{15}^{2}$. One of them, say $O_{15}^{1}$, has a nontrivial intersection with the subgraphs stabilized by $G_{3}$ and $G_{4}$ detailed in the previous section. On the other hand, $G_{0}$ has two orbits of size 21945 on the set of vertices $V_{\text {IvSh }}$, namely $O_{21945}^{1}$ and $O_{21945}^{2}$, on which it acts in the same way it acts on its 2-Sylow subgroups $2^{3}$. Therefore $G_{0 h}$ has at least one orbit of size 15 in each of those two $G_{0}$-orbits. Using the distance distribution map provided in Tables 11 and 12, we see that a point of $O_{1463}^{2}$ has neighbors in $O_{21945}^{1}$ and none in $O_{21945}^{2}$.

As we saw in the proof of Lemma 9.3, $\operatorname{Stab}_{G}[p, q]$ fixes two more points, say $r \in O_{1463}^{1}$ and $s \in O_{1463}^{3}$. By our previous developments, $r$ corresponds to the $2 B$-involution commuting with $p$ in $\operatorname{Aut}(G)$. Let $o$ be the vertex in $O_{21945}^{1}$ fixed 
by $G_{01 h} \cong 2^{3}$. Since $G_{01 h}$ is one of the 152 -Sylow subgroups of $G_{0 h}$, it belongs to a $G_{0 h}$-orbit of size 15, say $O_{15}^{2}$. At this point, we have identified 4 orbits under the action of $G_{0 h}: p, r, O_{15}^{1}$ and $O_{15}^{2}$. Notice that $G_{1 h}$ swaps $o$ and $p$. Let us now consider the subgroup $H:=\left\langle\operatorname{Stab}_{G}[p, r], \operatorname{Stab}_{G}\{p, o\}\right\rangle$. This subgroup acts transitively on those 32 points. By looking at the subgroup lattice of $G$, we see that common overgroups of subgroups of classes \#277 $\left(\mathrm{Stab}_{G}[p, r]\right)$ and \#527 $\left(\operatorname{Stab}_{G}\{p, o\}\right)$ could be in classes \#1, \#7, \#8, \#30,\#143, \#212 or \#213. Since $H$ acts transitively on 32 points, $H$ cannot be a subgroup of classes \#143, \#212 and \#213. It cannot be $G$ itself since $H$ is not transitive on all points. Since a subgroup of class \#30 is quasimaximal in $G$, it follows that $H$ is a subgroup of that class and $H=G_{h}$. We now define the $h$-element stabilized by $G_{h}$ to be the subgraph of the $H$-orbit of size 32 that we emphasized.

\subsubsection{Incidence relation}

We define an incidence relation $*$ on $X$ induced by maximal intersection, i.e. inclusion in all but two cases. More precisely, a 3-element $x$ is incident to a 4-element $y$ if and only if $|x \cap y|=3$, and a 4-element $y$ is incident to an $h$-element $z$ if and only if $|y \cap z|=4$.

According to Section 4 , the 3 -tuple $(X, *, \tau)$ defines a pregeometry $\Gamma$ of rank 5 over $I$.

\subsubsection{Firmness and residual connectedness}

The boolean lattice $L_{\mathrm{IvSh}}$ is generating, hence $\Gamma$ is residually connected by virtue of Lemma 3.1. Moreover $\Gamma$ is firm since all of its residues are.

\subsubsection{Flag-transitivity}

The group $G$ is transitive on the 0-elements of the pregeometry $\Gamma$. The stabilizer $J$ in $G$ of a 0 -element is isomorphic to $J_{1}$. The residue of a 0 -element is isomorphic to the pregeometry $\Gamma_{J_{1}}$ with $J$ acting flag-transitively on it. Let $C$ and $C^{\prime}$ be two chambers of $\Gamma$. By transitivity on the 0-elements, the 0-element $p$ of $C$ can be brought onto the 0 -element $q$ of $C^{\prime}$. Now the stabilizer of $q$ in $G$ is transitive on the flags incident to $q$. Hence there exists an element in $G$ that maps $C$ onto $C^{\prime}$. Therefore $\Gamma$ is a flag-transitive pregeometry, thus a flag-transitive geometry according to Section 4 . By uniqueness of the $\Gamma_{\mathrm{IvSh}}$ boolean lattices, $\Gamma$ is the $\Gamma_{\text {IvSh }}$ geometry.

This technique was used by Buekenhout to prove flag-transitivity in most of the geometries he got in his catalogue [6]. 


\subsection{Properties of $\Gamma$}

We just proved that $\Gamma$ is a firm, residually connected, flag-transitive geometry. Moreover $G$ acts primitively on the 0 -elements and the 4-elements of $\Gamma$ since the stabilizer of an element of each of those types is a maximal subgroup of $G$. Hence $\Gamma$ is weakly primitive, in the terminology of [8]. However $\Gamma$ is not residually weakly primitive since there exists a residue of $\Gamma$ that is not weakly primitive itself, namely the residue of a hyperline of $\Gamma$. This last property was proven by Buekenhout and Leemans in [12].

\subsubsection{The Buekenhout diagram of $\Gamma$}

Since the geometry $\Gamma$ is firm, residually connected and flag-transitive, we associate to it a Buekenhout diagram (see [10], Chapter 2, §3). This diagram is an amalgam of the diagrams of the residues of $\Gamma$. The orders can be computed by taking the index of the stabilizer of some element of each type in $G$. The resulting diagram is shown in Figure 19.

\section{More on $h$-elements}

In this section we provide a detailed study of the residue of an $h$-element $H$ in $\Gamma_{\text {IvSh }}$. The diagram $\Delta_{\text {IvSh }}$ together with the boolean lattice $L_{\text {IvSh }}$ of $\Gamma_{\text {IvSh }}$ yields the Buekenhout diagram of the residue $\Gamma_{H}$ of $H$ depicted in Figure 20. Since $\Gamma_{\text {IvSh }}$ is a flag-transitive, residually connected geometry, so is $\Gamma_{H}$. The truncation of this geometry on its elements of types in $\{0,1\}$ is a graph, that we call the underlying graph of $\Gamma_{H}$ and that we denote with ${ }_{\{0,1\}} \Gamma_{H}$. A 0 -element is now called a point and a 1-element an edge. The residue of a 2-element is obviously a hemi-icosahedron and the residue of a 3-element is a tetrahedron.

Let us describe the structure of ${ }_{\{0,1\}} \Gamma_{H}$. By flag-transitivity of $\Gamma_{H}$, the truncation $\{0,1\} \Gamma_{H}$ is flag-transitive, i.e. arc-transitive in the terminology of graph theory. Every vertex has degree 15, there are 32 vertices and 240 edges. Since $\Gamma_{H}$ is residually connected, $\{0,1\} \Gamma_{H}$ is connected. The group $4 \cdot 2^{4}: A_{5}$ acts flag-transitively on ${ }_{\{0,1\}} \Gamma_{H}$ with a kernel of size 2 , whose quotient is $2^{5}: A_{5}$. This quotient has a central involution which induces a pairing of the vertices of $\{0,1\} \Gamma_{H}$. The vertex paired with $p$ through the central involution is called the opposite of $p$ and is denoted by $p^{o p}$. Hence the distance distribution map of the graph ${ }_{\{0,1\}} \Gamma_{H}$ is endowed with a central symmetry and can be depicted as in Figure 21. There remains to determine the unknowns $a$ and $b$.

Lemma 10.1. The distance distribution map of ${ }_{\{0,1\}} \Gamma_{H}$ is as depicted in Figure 22. 


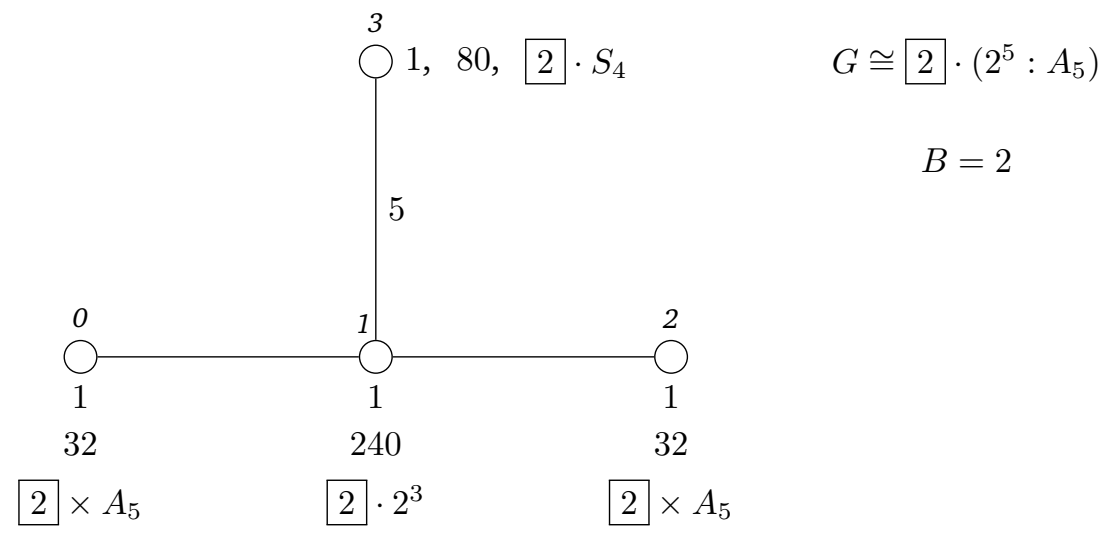

Figure 20: The residue of a hyperline in $\Gamma_{\text {IvSh }}$

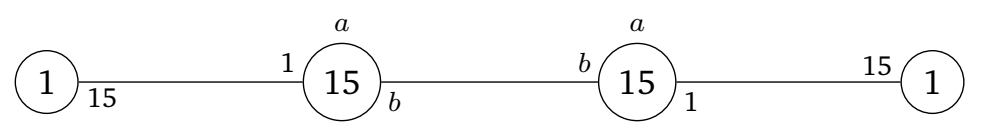

Figure 21: The distance distribution map of the underlying graph of a hyperline

Proof. Since the residue of a 2 -element is a hemi-icosahedron, the $\{0,1\}$-shadow of a 2-element in $\Gamma_{H}$ is a clique $K_{6}$ of 6 vertices. The diagram shows that there are 6 elements of type 2 incident with each point. Therefore every vertex of $\{0,1\} \Gamma_{H}$ lies in 6 cliques $K_{6}$. Hence the neighborhood $p^{\perp}$ of any vertex $p$ of $\{0,1\} \Gamma_{H}$ contains 6 cliques of size 5 . By flag-transitivity, every vertex of $p^{\perp}$ is contained in the same number $x$ of cliques $K_{5}$. By double counting, we obtain

$$
5 \times 6=15 x \Longleftrightarrow x=2 .
$$

We deduce that $a \geq 8$ and thus $b \leq 6$. Every edge of ${ }_{\{0,1\}} \Gamma_{H}$ is contained in exactly 2 cliques of size 6 . Given two adjacent vertices $v \in p^{\perp}$ and $w \in p^{o p \perp}$, we conclude that they must have at least 8 common neighbors, and thus $b \geq$ $\frac{8}{2}+1=5$. Finally, we observe that $p, w \in v^{\perp}$ are at distance 2 in ${ }_{\{0,1\}} \Gamma_{H}$. By flag-transitivity, we conlude that there exists a vertex in $p^{\perp}$ adjacent to $w$ but not adjacent to $v$. Hence $w$ has a sixth neighbor in $p^{\perp}$ and $b=6, a=8$.

Now we proceed with the recognition of the graph ${ }_{\{0,1\}} \Gamma_{H}$. First of all, let us introduce some terminology and a surprising situation. The $n$-halved cube is a graph of $2^{n-1}$ vertices consisting of one of the two connected components of the graph of vertices at distance 2 in the $n$-cube graph. In [23], Imrich, Klavzar and Vesel provide a characterization of halved cube graphs as follows. 
Lemma 10.2 ([23]). Let $n \geq 5$. The $n$-halved cube graph $\mathcal{Q}_{n}$ is the only connected, $\left(\begin{array}{l}n \\ 2\end{array}\right)$-regular graph on $2^{n-1}$ vertices in which every edge is in two $n$-cliques and no two $n$-cliques intersect in a vertex.

We use Lemma 10.2 to characterize ${ }_{\{0,1\}} \Gamma_{H}$ in Theorem 10.4. During that process, we also make use of Lemma 10.3 due to Harary [21].

Lemma 10.3 ([21, Theorem 8.4]). A graph is a line graph if and only if its edges can be partitioned into complete subgraphs in such a way that no vertex lies in more than two of the subgraphs.

Given a line graph $\mathcal{L}(\mathcal{G})$ and a partition $\mathcal{P}$ as in Lemma 10.3, we recover the original graph $G$ as follows: the vertex set of $G$ is the set $\mathcal{P}$; two vertices of $G$ are now joined by an edge if and only if the corresponding cliques share a vertex in $\mathcal{L}(\mathcal{G})$.

Theorem 10.4. The underlying graph ${ }_{\{0,1\}} \Gamma_{H}$ of a hyperline $H$ is isomorphic to the 6-halved cube.

Proof. The purpose of the proof is to show that ${ }_{\{0,1\}} \Gamma_{H}$ satisfies the hypotheses of Lemma 10.2. Notice that ${ }_{\{0,1\}} \Gamma_{H}$ has $2^{5}=2^{6-1}$ vertices and that it is regular of valency $\left(\begin{array}{l}6 \\ 2\end{array}\right)=15$. Let $p$ be any vertex of ${ }_{\{0,1\}} \Gamma_{H}$ and let $p^{\perp}$ be its neighborhood. As we observe in the proof of Lemma 10.1, every vertex of $p^{\perp}$ sits in exactly two 5 -cliques. We now apply Lemma 10.3 to conclude that $p^{\perp}$ is the line graph of some graph. We recover that graph by applying the construction described after Lemma 10.3 and we see that the induced subgraph on $p^{\perp}$ is isomorphic to the line graph of a 6-clique. Moreover every edge of $\{0,1\} \Gamma_{H}$ sits in exactly two 6-cliques. It is also obvious that two 6-cliques cannot intersect in a single vertex. We finish the proof by applying Lemma 10.2.

The full automorphism group of ${ }_{\{0,1\}} \Gamma_{H} \cong \mathcal{Q}_{6}$ is isomorphic to $2^{5}: S_{6}$. Let us mention that the neighborhood of any point in ${ }_{\{0,1\}} \Gamma_{H}$ is isomorphic to the line graph of a complete graph of 6 vertices with $S_{6}$ acting. Observe moreover that this graph of 15 vertices is also the complement of the collinearity graph of the generalized quadrangle of order $(2,2)$ with $S p_{4}(2) \cong S_{6}$ acting. It is also the graph of hyperbolic lines of that generalized quadrangle.

Finally Theorem 10.5 shows that our $h$-elements deserve the name hyperline.

Theorem 10.5. Through any pair of opposite points, there is a unique hyperline.

Proof. Let $p$ be any vertex of $\mathcal{G}_{\text {IvSh }}$. Then there are 1463 opposites to $p$ and there are 1463 hyperlines through $p$. Since there is one opposite to $p$ in each hyperline, any pair of opposite points provides a unique hyperline. 


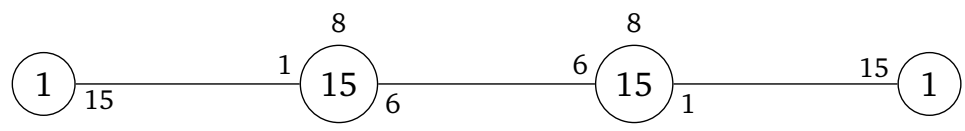

Figure 22: The distance distribution map of the underlying graph of a hyperline

\section{Computational resources}

Generators for each maximal parabolic subgroup of $\Gamma_{\text {IvSh }}$ are available upon request to the authors. They are given as permutations of $\mathrm{O}^{\prime} \mathrm{N}$ in its representation on 122760 points. They were obtained following the process of the proof of Theorem 8.1 in MAGMA [3].

\section{Acknowledgements}

We thank Antonio Pasini, Theo Grundhöfer, Karl Strambach and an anonymous referee for their friendly and outstanding help as well as useful remarks on an earlier submitted version of this paper. Many improvements were possible using those comments. We also thank Dimitri Leemans for useful remarks regarding Section 4.3. Finally our thanks go to Alexander Ivanov who provided a copy of the original paper [24].

This research was conducted while Thomas Connor was funded by the Fonds pour la Formation à la Recherche dans l'Industrie et dans l'Agriculture, for which he is grateful.

\section{Appendix}

We provide subgroup lattices of various groups appearing in this work as well as the distance distribution map of the graph $\mathcal{G}_{\mathrm{IVSh}}$.

\begin{tabular}{||c|l|c|c|l|l||}
\hline Nr. & Structure & Order & Length & Maximal Subgroups & Minimal Overgroups \\
\hline 1 & $\mathrm{M}_{11}$ & 7920 & 1 & $2(11), 3(12), 5(55), 6(66), 13(165)$ & \\
\hline 2 & $\mathrm{M}_{10}$ & 720 & 11 & $4,7(10), 19(36), 22(45)$ & 1 \\
\hline 3 & $L_{2}(11)$ & 660 & 12 & $10(11), 11(11), 12(12), 23(55)$ & 1 \\
\hline 4 & $A_{6}$ & 360 & 11 & $11(12), 14(10), 17(30)$ & 2 \\
\hline 5 & $M_{9}: 2$ & 144 & 55 & $8,7,9,22(9)$ & 1 \\
\hline 6 & $S_{5}$ & 120 & 66 & $10,17(5), 19(6), 23(10)$ & 1 \\
\hline 7 & $M_{9}$ & 72 & 55 & $14(2), 15,28(9)$ & $2(2), 5$ \\
\hline 8 & $3: S_{3} \cdot 2 \cdot 2$ & 72 & 55 & $15,29(9)$ & 5 \\
\hline 9 & $3: S_{3} \cdot 2: 2$ & 72 & 55 & $16(2), 15,30(9)$ & $3(2), 6$ \\
\hline 10 & $A_{5}$ & 60 & 66 & $24(5), 26(6), 31(10)$ & 3,4 \\
\hline 11 & $A_{5}$ & 60 & 132 & $24(5), 26(6), 32(10)$ & 3 \\
\hline 12 & $11: 5$ & 55 & 144 & $25,34(11)$ & 1 \\
\hline 13 & $Q_{8}: S_{3}$ & 48 & 165 & $18,22(3), 23(4)$ & continued on next page \\
\hline \multicolumn{7}{|l}{} \\
\hline
\end{tabular}




\begin{tabular}{|c|c|c|c|c|c|}
\hline \multicolumn{6}{|c|}{ continued from previous page } \\
\hline $\mathrm{Nr}$. & Structure & Order & Length & Maximal Subgroups & Minimal Overgroups \\
\hline 14 & $3: S_{3} \cdot 2$ & 36 & 110 & $20,35(9)$ & 4,7 \\
\hline 15 & $3: S_{3} \cdot 2$ & 36 & 55 & $20,35(9)$ & $7,8,9$ \\
\hline 16 & $S_{3} \times S_{3}$ & 36 & 110 & $20,21(2), 23(6)$ & 9 \\
\hline 17 & $2^{2}: S_{3}$ & 24 & 330 & $24,30(3), 32(4)$ & 4,6 \\
\hline 18 & $Q_{8}: 3$ & 24 & 165 & $28,33(4)$ & 13 \\
\hline 19 & $D_{10} \cdot 2$ & 20 & 396 & 26,35 (5) & 2,6 \\
\hline 20 & $3: S_{3}$ & 18 & 55 & $27,32(12)$ & $14(2), 15,16(2)$ \\
\hline 21 & $S_{3} \times 3$ & 18 & 220 & $27,31,33(3)$ & 16 \\
\hline 22 & $Q_{8}: 2$ & 16 & 495 & $30,29,28$ & $2,5,13$ \\
\hline 23 & $D_{12}$ & 12 & 660 & $32,33,31,36(3)$ & $3,6,13,16$ \\
\hline 24 & $A_{4}$ & 12 & 330 & $36,37(4)$ & $10,11(2), 17$ \\
\hline 25 & 11 & 11 & 144 & 39 & 12 \\
\hline 26 & $D_{10}$ & 10 & 396 & $34,38(5)$ & $10,11(2), 19$ \\
\hline 27 & $3^{2}$ & 9 & 55 & $37(4)$ & $20,21(4)$ \\
\hline 28 & $Q_{8}$ & 8 & 165 & $35(3)$ & $7(3), 18,22(3)$ \\
\hline 29 & 8 & 8 & 495 & 35 & 8,22 \\
\hline 30 & $D_{8}$ & 8 & 495 & $36(2), 35$ & $9,17(2), 22$ \\
\hline 31 & $S_{3}$ & 6 & 220 & $37,38(3)$ & $10(3), 21,23(3)$ \\
\hline 32 & $S_{3}$ & 6 & 660 & $37,38(3)$ & $11(2), 17(2), 20,23$ \\
\hline 33 & 6 & 6 & 660 & 37,38 & $18,21,23$ \\
\hline 34 & 5 & 5 & 396 & 39 & $12(4), 26$ \\
\hline 35 & 4 & 4 & 495 & 38 & $14(2), 15,19(4), 28,29,30$ \\
\hline 36 & $2^{2}$ & 4 & 330 & $38(3)$ & $23(6), 24,30(3)$ \\
\hline 37 & 3 & 3 & 220 & 39 & $24(6), 27,31,32(3), 33(3)$ \\
\hline 38 & 2 & 2 & 165 & 39 & $26(12), 31(4), 32(12), 33(4), 35(3), 36(6)$ \\
\hline 39 & 1 & 1 & 1 & & $25(144), 34(396), 37(220), 38(165)$ \\
\hline
\end{tabular}

Table 4: Subgroup lattice of $\mathrm{M}_{11}$

\begin{tabular}{|c|c|c|c|c|c|}
\hline Nr. & Structure & Order & Length & Maximal Subgroups & Minimal Overgroups \\
\hline 1 & $\mathrm{~J}_{1}$ & 175560 & 1 & $\begin{array}{l}2 \text { (266), } 3(1045), 4(1463), 5(1540), \\
6(1596), 7(2926), 13(4180)\end{array}$ & \\
\hline 2 & $L_{2}(11)$ & 660 & 266 & $8(11), 9(11), 12(12), 25(55)$ & 1 \\
\hline 3 & $2^{3}: 7: 3$ & 168 & 1045 & $11,18(7), 20(8)$ & 1 \\
\hline 4 & $A_{5} \times 2$ & 120 & 1463 & $9,18(5), 21(6), 25(10)$ & 1 \\
\hline 5 & $19: 3: 2$ & 114 & 1540 & $10,14,33(19)$ & 1 \\
\hline 6 & $11: 5: 2$ & 110 & 1596 & $12,19,28(11)$ & 1 \\
\hline 7 & $D_{10} \times S_{3}$ & 60 & 2926 & $15,16,17,21(3), 25(5)$ & 1 \\
\hline 8 & $A_{5}$ & 60 & 2926 & $26(5), 29(6), 34(10)$ & 2 \\
\hline 9 & $A_{5}$ & 60 & 1463 & $26(5), 29(6), 35(10)$ & $2(2), 4$ \\
\hline 10 & $19: 3$ & 57 & 1540 & $22,38(19)$ & 5 \\
\hline 11 & $2^{3}: 7$ & 56 & 1045 & $31,32(8)$ & 3 \\
\hline 12 & $11: 5$ & 55 & 1596 & $27,36(11)$ & $2(2), 6$ \\
\hline 13 & $7: 3: 2$ & 42 & 4180 & $20,24,33(7)$ & 1 \\
\hline 14 & $D_{38}$ & 38 & 1540 & $22,39(19)$ & 5 \\
\hline 15 & $S_{3} \times 5$ & 30 & 2926 & $23,28(3), 35$ & 7 \\
\hline 16 & $15: 2$ & 30 & 2926 & $23,30,33(5)$ & 7 \\
\hline 17 & $D_{30}$ & 30 & 2926 & $23,29(3), 34(5)$ & 7 \\
\hline 18 & $A_{4} \times 2$ & 24 & 7315 & $26,31,33(4)$ & 3,4 \\
\hline 19 & $D_{22}$ & 22 & 1596 & $27,39(11)$ & 6 \\
\hline 20 & $7: 3$ & 21 & 4180 & $32,38(7)$ & $3(2), 13$ \\
\hline 21 & $D_{20}$ & 20 & 8778 & $29,28,30,37(5)$ & 4,7 \\
\hline 22 & 19 & 19 & 1540 & 40 & 10,14 \\
\hline 23 & 15 & 15 & 2926 & 36,38 & $15,16,17$ \\
\hline 24 & $D_{14}$ & 14 & 4180 & $32,39(7)$ & 13 \\
\hline 25 & $D_{12}$ & 12 & 14630 & $35,33,34,37(3)$ & $2,4,7$ \\
\hline 26 & $A_{4}$ & 12 & 7315 & $37,38(4)$ & $8(2), 9,18$ \\
\hline 27 & 11 & 11 & 1596 & 40 & 12,19 \\
\hline 28 & 10 & 10 & 8778 & 36,39 & $6(2), 15,21$ \\
\hline 29 & $D_{10}$ & 10 & 8778 & $36,39(5)$ & $8(2), 9,17,21$ \\
\hline 30 & $D_{10}$ & 10 & 2926 & $36,39(5)$ & $16,21(3)$ \\
\hline 31 & $2^{3}$ & 8 & 1045 & $37(7)$ & $11,18(7)$ \\
\hline 32 & 7 & 7 & 4180 & 40 & $11(2), 20,24$ \\
\hline 33 & 6 & 6 & 14630 & 38,39 & $5(2), 13(2), 16,18(2), 25$ \\
\hline 34 & $S_{3}$ & 6 & 14630 & $38,39(3)$ & $8(2), 17,25$ \\
\hline 35 & $S_{3}$ & 6 & 2926 & $38,39(3)$ & $9(5), 15,25(5)$ \\
\hline 36 & 5 & 5 & 2926 & 40 & $12(6), 23,28(3), 29(3), 30$ \\
\hline 37 & $2^{2}$ & 4 & 7315 & $39(3)$ & $21(6), 25(6), 26,31$ \\
\hline 38 & 3 & 3 & 2926 & 40 & 10 (10), 20 (10), $23,26(10), 33(5), 34(5), 35$ \\
\hline
\end{tabular}




\begin{tabular}{|c|c|c|c|c|c|}
\hline \multicolumn{6}{|c|}{ continued from previous page } \\
\hline Nr. & Structure & Order & Length & Maximal Subgroups & Minimal Overgroups \\
\hline 39 & 2 & 2 & 1463 & 40 & $\begin{array}{l}14(20), 19(122), 24(20), 28(6), 29(30), 30 \\
(10), 33(10), 34(30), 35(6), 37(15)\end{array}$ \\
\hline 40 & 1 & 1 & 1 & & $\begin{array}{l}22(1540), 27(1596), 32(4180), 36(2926), 38 \\
(2926), 39(1463)\end{array}$ \\
\hline
\end{tabular}

Table 5: Subgroup lattice of $\mathrm{J}_{1}$

\begin{tabular}{|c|c|c|c|c|c|}
\hline Nr. & Structure & Order & Length & Maximal Subgroups & Minimal Overgroups \\
\hline 1 & $S_{5} \times 2$ & 240 & 1 & $2,3,4,6(5), 7(6), 8(10)$ & \\
\hline 2 & $S_{5}$ & 120 & 1 & $5,9(5), 12(6), 16(10)$ & 1 \\
\hline 3 & $S_{5}$ & 120 & 1 & $5,10(5), 13(6), 17(10)$ & 1 \\
\hline 4 & $A_{5} \times 2$ & 120 & 1 & $5,11(5), 14(6), 18(10)$ & 1 \\
\hline 5 & $A_{5}$ & 60 & 1 & $19(5), 24(6), 34(10)$ & $2,3,4$ \\
\hline 6 & $S$ & 48 & 5 & $10,9,11,15(3), 20(4)$ & 1 \\
\hline 7 & $D_{10} \cdot 2 \times 2$ & 40 & 6 & $13,14,12,27(5)$ & 1 \\
\hline 8 & $S_{3} \times 2^{2}$ & 24 & 10 & $21,20,22,16,17,23,18,28$ (3) & 1 \\
\hline 9 & $S_{4}$ & 24 & 5 & $19,29(3), 35(4)$ & 2,6 \\
\hline 10 & $\frac{4}{S_{4}}$ & 24 & 5 & $19,30(3), 36(4)$ & 3,6 \\
\hline 11 & $A_{4} \times 2$ & 24 & 5 & $19,31,37(4)$ & 4,6 \\
\hline 12 & $D_{10} \cdot 2$ & 20 & 6 & $24,42(5)$ & 2,7 \\
\hline 13 & $D_{10} \cdot 2$ & 20 & 6 & $24,43(5)$ & 3,7 \\
\hline 14 & $D_{20}$ & 20 & 6 & $25,26,24,44(5)$ & 4,7 \\
\hline 15 & $D_{8} \times 2$ & 16 & 15 & $29,32,28,31,33,27,30$ & 6 \\
\hline 16 & $D_{12}$ & 12 & 10 & $34,35,38,45(3)$ & 2,8 \\
\hline 17 & $D_{12}$ & 12 & 10 & $39,36,34,46(3)$ & 3,8 \\
\hline 18 & $D_{12}$ & 12 & 10 & $40,37,34,44(3)$ & 4,8 \\
\hline 19 & $A_{4}$ & 12 & 5 & $47,51(4)$ & $5,9,10,11$ \\
\hline 20 & $D_{12}$ & 12 & 10 & $37,36,35,48(3)$ & $6(2), 8$ \\
\hline 21 & $D 12$ & 12 & 10 & $40,39,35,49(3)$ & 8 \\
\hline 22 & $D_{12}$ & 12 & 10 & $36,40,38,49(3)$ & 8 \\
\hline 23 & $2 \times 6$ & 12 & 10 & $37,39,38,48$ & 8 \\
\hline 24 & $D_{10}$ & 10 & 6 & $41,52(5)$ & $5,12,13,14$ \\
\hline 25 & $D_{10}$ & 10 & 6 & $41,53(5)$ & 14 \\
\hline 26 & 10 & 10 & 6 & 41,54 & 14 \\
\hline 27 & $2 \times 4$ & 8 & 15 & $42,43,44$ & $7(2), 15$ \\
\hline 28 & $2^{3}$ & 8 & 15 & $45,44,48(2), 49(2), 46$ & $8(2), 15$ \\
\hline 29 & $D_{8}$ & 8 & 15 & $45,42,47$ & 9,15 \\
\hline 30 & $D_{8}$ & 8 & 15 & $46,43,47$ & 10,15 \\
\hline 31 & $2^{3}$ & 8 & 5 & $44(3), 50(3), 47$ & $11,15(3)$ \\
\hline 32 & $D_{8}$ & 8 & 15 & $46,42,50$ & 15 \\
\hline 33 & $D_{8}$ & 8 & 15 & $45,50,43$ & 15 \\
\hline 34 & $S_{3}$ & 6 & 10 & $51,52(3)$ & $5,16,17,18$ \\
\hline 35 & $\frac{s}{S_{3}}$ & 6 & 10 & $51,55(3)$ & $9(2), 16,20,21$ \\
\hline 36 & $S_{3}$ & 6 & 10 & $51,56(3)$ & $10(2), 17,20,22$ \\
\hline 37 & 6 & 6 & 10 & 51,54 & $11(2), 18,20,23$ \\
\hline 38 & 6 & 6 & 10 & 51,55 & $16,22,23$ \\
\hline 39 & 6 & 6 & 10 & 51,56 & $17,21,23$ \\
\hline 40 & $S_{3}$ & 6 & 10 & $51,53(3)$ & $18,21,22$ \\
\hline 41 & 5 & 5 & 6 & 57 & $24,25,26$ \\
\hline 42 & 4 & 4 & 15 & 52 & $12(2), 27,29,32$ \\
\hline 43 & 4 & 4 & 15 & 52 & $13(2), 27,30,33$ \\
\hline 44 & $2^{2}$ & 4 & 15 & $52,53,54$ & $14(2), 18(2), 27,28,31$ \\
\hline 45 & $2^{2}$ & 4 & 15 & $55(2), 52$ & $16(2), 28,29,33$ \\
\hline 46 & $2^{2}$ & 4 & 15 & $52,56(2)$ & $17(2), 28,30,32$ \\
\hline 47 & $2^{2}$ & 4 & 5 & $52(3)$ & $19,29(3), 30(3), 31$ \\
\hline 48 & $2^{2}$ & 4 & 10 & $55,54,56$ & $20(3), 23,28(3)$ \\
\hline 49 & $2^{2}$ & 4 & 30 & $55,53,56$ & $21,22,28$ \\
\hline 50 & $2^{2}$ & 4 & 15 & $53(2), 52$ & $31,32,33$ \\
\hline 51 & 3 & 3 & 10 & 57 & $19(2), 34,35,36,37,38,39,40$ \\
\hline 52 & 2 & 2 & 15 & 57 & $24(2), 34(2), 42,43,44,45,46,47,50$ \\
\hline 53 & 2 & 2 & 15 & 57 & $25(2), 40(2), 44,49(2), 50(2)$ \\
\hline 54 & 2 & 2 & 1 & 57 & $26(6), 37(10), 44(15), 48(10)$ \\
\hline 55 & 2 & 2 & 10 & 57 & $35(3), 38,45(3), 48,49(3)$ \\
\hline 56 & 2 & 2 & 10 & 57 & $36(3), 39,46(3), 48,49(3)$ \\
\hline 57 & 1 & 1 & 1 & & $\begin{array}{l}41(6), 51(10), 52(15), 53(15), 54,55(10), \\
56(10)\end{array}$ \\
\hline
\end{tabular}

Table 6: Subgroup lattice of $2 \times S_{5}$ 


\begin{tabular}{||l|l|c|c|l|l||}
\hline Nr. & Structure & Order & Length & Maximal Subgroups & Minimal Overgroups \\
\hline 1 & $L_{2}(11)$ & 660 & 1 & $2(11), 3(11), 4(12), 5(55)$ & \\
\hline 2 & $A_{5}$ & 60 & 11 & $6(5), 8(6), 9(10)$ & 1 \\
\hline 3 & $A_{5}$ & 60 & 11 & $6(5), 8(6), 10(10)$ & 1 \\
\hline 4 & $11: 5$ & 55 & 12 & $7,12(11)$ & 1 \\
\hline 5 & $D_{12}$ & 12 & 55 & $11,9,10,13(3)$ & 1 \\
\hline 6 & $A_{4}$ & 12 & 55 & $13,14(4)$ & 2,3 \\
\hline 7 & 11 & 11 & 12 & 16 & 4 \\
\hline 8 & $D_{10}$ & 10 & 66 & $12,15(5)$ & 2,3 \\
\hline 9 & $S_{3}$ & 6 & 55 & $14,15(3)$ & $2(2), 5$ \\
\hline 10 & $S_{3}$ & 6 & 55 & $14,15(3)$ & $3(2), 5$ \\
\hline 11 & 6 & 6 & 55 & 14,15 & 5 \\
\hline 12 & 5 & 5 & 66 & 16 & $4(2), 8$ \\
\hline 13 & 2 & 4 & 55 & $15(3)$ & $5(3), 6$ \\
\hline 14 & 3 & 3 & 55 & 16 & $6(4), 9,10,11$ \\
\hline 15 & 2 & 2 & 55 & 16 & $8(6), 9(3), 10(3), 11,13(3)$ \\
\hline 16 & 1 & 1 & 1 & & $7(12), 12(66), 14(55), 15(55)$ \\
\hline
\end{tabular}

Table 7: Subgroup lattice of $L_{2}(11)$

\begin{tabular}{|c|c|c|c|c|c|}
\hline Nr. & Structure & Order & Length & Maximal Subgroups & Minimal Overgroups \\
\hline 1 & $A_{5} \times S_{3}$ & 360 & 1 & $2,3(3), 4(5), 5(6), 7(10)$ & \\
\hline 2 & $A_{5} \times 3$ & 180 & 1 & $6,8(5), 9(6), 15(10)$ & 1 \\
\hline 3 & $A_{5} \times 2$ & 120 & 3 & $6,12(5), 14(6), 19(10)$ & 1 \\
\hline 4 & $A_{4} \times S_{3}$ & 72 & 5 & $8,13,12(3), 16(4)$ & 1 \\
\hline 5 & $D_{10} \times S_{3}$ & 60 & 6 & $9,10,11,14(3), 20(5)$ & 1 \\
\hline 6 & $A_{5}$ & 60 & 1 & $21(5), 25(6), 30(10)$ & $2,3(3)$ \\
\hline 7 & $S_{3} \times S_{3}$ & 36 & 10 & $17,16,15,19(3), 20(3)$ & 1 \\
\hline 8 & $A_{4} \times 3$ & 36 & 5 & $22(2), 21,23,28(4)$ & 2,4 \\
\hline 9 & $15: 2$ & 30 & 6 & $18,25,31(5)$ & 2,5 \\
\hline 10 & $S_{3} \times 5$ & 30 & 6 & $18,26(3), 32$ & 5 \\
\hline 11 & $D_{30}$ & 30 & 6 & $18,27(3), 33(5)$ & 5 \\
\hline 12 & $A_{4} \times 2$ & 24 & 15 & $21,29,34(4)$ & 3,4 \\
\hline 13 & $S_{3} \times 2^{2}$ & 24 & 5 & $20(3), 24(3), 23,29(3)$ & 4 \\
\hline 14 & $D_{20}$ & 20 & 18 & $27,25,26,38(5)$ & 3,5 \\
\hline 15 & $S_{3} \times 3$ & 18 & 10 & $28,30,31(3)$ & 2,7 \\
\hline 16 & $S_{3} \times 3$ & 18 & 10 & $28,32,34(3)$ & $4(2), 7$ \\
\hline 17 & $3: S_{3}$ & 18 & 10 & $28,35(3), 36(6), 33(3)$ & 7 \\
\hline 18 & 15 & 15 & 6 & 37,41 & $9,10,11$ \\
\hline 19 & $D_{12}$ & 12 & 30 & $30,35,34,38(3)$ & 3,7 \\
\hline 20 & $D_{12}$ & 12 & 15 & $33,31,32,38(3)$ & $5(2), 7(2), 13$ \\
\hline 21 & $A_{4}$ & 12 & 5 & $39,42(4)$ & $6,8,12(3)$ \\
\hline 22 & $A_{4}$ & 12 & 10 & $39,43(4)$ & 8 \\
\hline 23 & $2 \times 6$ & 12 & 5 & $31(3), 39$ & 8,13 \\
\hline 24 & $D_{12}$ & 12 & 15 & $33(2), 31,40(3)$ & 13 \\
\hline 25 & $D_{10}$ & 10 & 6 & $37,44(5)$ & $6,9,14(3)$ \\
\hline 26 & 10 & 10 & 18 & 37,45 & 10,14 \\
\hline 27 & $D_{10}$ & 10 & 18 & $37,46(5)$ & 11,14 \\
\hline 28 & $3^{2}$ & 9 & 10 & $41,43(2), 42$ & $8(2), 15,16,17$ \\
\hline 29 & $2^{3}$ & 8 & 15 & $38(3), 40(3), 39$ & 12,13 \\
\hline 30 & $S_{3}$ & 6 & 10 & $42,44(3)$ & $6,15,19(3)$ \\
\hline 31 & $\frac{6}{6}$ & 6 & 15 & 41,44 & $9(2), 15(2), 20,23,24$ \\
\hline 32 & $S_{3}$ & 6 & 1 & $41,45(3)$ & $10(6), 16(10), 20(15)$ \\
\hline 33 & $S_{3}$ & 6 & 15 & $41,46(3)$ & $11(2), 17(2), 20,24(2)$ \\
\hline 34 & 6 & 6 & 30 & 42,45 & $12(2), 16,19$ \\
\hline 35 & $S_{3}$ & 6 & 30 & $42,46(3)$ & 17,19 \\
\hline 36 & $S_{3}$ & 6 & 60 & $43,46(3)$ & 17 \\
\hline 37 & 5 & 5 & 6 & 47 & $18,25,26(3), 27(3)$ \\
\hline 38 & $2^{2}$ & 4 & 45 & $45,46,44$ & $14(2), 19(2), 20,29$ \\
\hline 39 & $2^{2}$ & 4 & 5 & $44(3)$ & $21,22(2), 23,29(3)$ \\
\hline 40 & $2^{2}$ & 4 & 45 & $46(2), 44$ & 24,29 \\
\hline 41 & 3 & 3 & 1 & 47 & $18(6), 28(10), 31(15), 32,33(15)$ \\
\hline 42 & 3 & 3 & 10 & 47 & $21(2), 28,30,34(3), 35(3)$ \\
\hline 43 & 3 & 3 & 20 & 47 & $22(2), 28,36(3)$ \\
\hline 44 & 2 & 2 & 15 & 47 & $25(2), 30(2), 31,38(3), 39,40(3)$ \\
\hline 45 & 2 & 2 & 3 & 47 & $26(6), 32,34(10), 38(15)$ \\
\hline 46 & 2 & 2 & 45 & 47 & $27(2), 33,35(2), 36(4), 38,40(2)$ \\
\hline 47 & 1 & 1 & 1 & & $\begin{array}{l}37(6), 41,42(10), 43(20), 44(15), 45(3), \\
46(45)\end{array}$ \\
\hline
\end{tabular}

Table 8: Subgroup lattice of $A_{5} \times S_{3}$ 


\begin{tabular}{|c|c|c|c|c|c|}
\hline Nr. & Structure & $\begin{array}{l}\text { Order } \\
\end{array}$ & Length & $\begin{array}{l}\text { Maximal Subgroups } \\
\end{array}$ & $\begin{array}{l}\text { Minimal Overgroups } \\
\end{array}$ \\
\hline 1 & $\mathrm{O}^{\prime} \mathrm{N}$ & 460815505920 & 1 & $\begin{array}{lr}2(122760), & 3(122760), \\
6(2624832), & 7(2857239), \\
11(17778376), & 12(17778376), \\
15(30968784), & 16(30968784), \\
21(42858585), & 23(58183776), \\
24(58183776), & \\
43(182863296), & \\
44(182863296)\end{array}$ & \\
\hline 6 & $\mathrm{~J}_{1}$ & 175560 & 2624832 & $\begin{array}{ll}119(266), & 237(1045), \\
277(1463), & 285(1540), \\
286(1596), & 366(2926), \\
397(4180) & \\
\end{array}$ & 1 \\
\hline 23 & $\mathrm{M}_{11}$ & 7920 & 58183776 & $\begin{array}{l}102(11), \quad 119(12), \quad 252(55), \\
278(66), 382(165)\end{array}$ & 1 \\
\hline 30 & $4 \cdot 2^{4}: A_{5}$ & 3840 & 120004038 & $\begin{array}{lll}97(5), & 133(6), & 156(10), \\
208(16), & 209(16), & 210(16), \\
211(16) & & \\
\end{array}$ & 8 \\
\hline 119 & $L_{2}(11)$ & 660 & 698205312 & 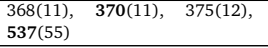 & $6,23,24$ \\
\hline 166 & $A_{5} \times S_{3}$ & 360 & 1280043072 & $\begin{array}{l}232,282(3), 335(5), 366(6), \\
407(10)\end{array}$ & 48,86 \\
\hline 212 & $2 \times S_{5}$ & 240 & 960032304 & $\begin{array}{l}278(2), 277,393(5), 402(6), \\
478(10)\end{array}$ & 143 \\
\hline 277 & $2 \times A_{5}$ & 120 & 960032304 & $370,482(5), 494(6), 537(10)$ & $6(4), 210,212,213$ \\
\hline 278 & $S_{5}$ & 120 & 1920064608 & $370,472(5), 491(6), 537(10)$ & $23(2), 212$ \\
\hline 282 & $2 \times A_{5}$ & 120 & 1920064608 & $371,485(5), 494(6), 537(10)$ & $104,165(2), 166(2), 209$ \\
\hline 366 & $S_{3} \times D_{10}$ & 60 & 7680258432 & $464,465,463,494(3), \mathbf{5 3 7}(5)$ & $6,165,166,230$ \\
\hline 370 & $A_{5}$ & 60 & 960032304 & $545(5), 550(6), 573(10)$ & $119(8), 277,278(2), 279(2)$ \\
\hline 382 & $2 \cdot S_{4}$ & 48 & 4800161520 & $477,514(3), 537(4)$ & $23(2), 229,296$ \\
\hline 407 & $S_{3} \times S_{3}$ & 36 & 6400215360 & $497(2), 502, \mathbf{5 3 7 ( 6 )}$ & $166(2), 253(2), 287(2), 330$ \\
\hline 478 & $3: 2^{3}$ & 24 & 3200107680 & 537(3), 539(3), 542, 568(3) & $\begin{array}{l}110(3), \quad 212(3), \quad 335, \quad 337, \\
386(3)\end{array}$ \\
\hline 527 & $Q_{8}: 2$ & 16 & 3600121140 & $567,562,564(2), 565(2), 568$ & $\begin{array}{l}393(4), 394(4), 447(2), 449(2), \\
455\end{array}$ \\
\hline 537 & $D_{12}$ & 12 & 9600323040 & $573,572,571,578(3)$ & $\begin{array}{l}119(4), 277,278(2), 279(2), \\
282(2), 366(4), 382(2), 383(2), \\
387(2), 406(4), 407(4), 418, \\
478,479,481\end{array}$ \\
\hline 564 & $D_{8}$ & 8 & 3600121140 & $578(2), 575$ & $\begin{array}{l}330(8), 472(8), 504,505(2), \\
514(2), 526,527(2), 530(2), \\
531\end{array}$ \\
\hline 567 & $2^{3}$ & 8 & 42858585 & 578(7) & $\begin{array}{l}\begin{array}{l}374(64), \\
527(84)\end{array} \\
\end{array}$ \\
\hline 568 & $2^{3}$ & 8 & 1200040380 & 578(3), 577(4) & $\begin{array}{l}\text { 478(8), 479(8), 485(4), 521, } \\
522(6), 523(6), 527(3), 531(3)\end{array}$ \\
\hline 573 & $S_{3}$ & 6 & 213340512 & $579, \mathbf{5 8 0 ( 3 )}$ & $\begin{array}{lll}370(45), & 371(10), & 465(36), \\
480(10), & 496(20), & 497(20), \\
500,537(45) & \\
\end{array}$ \\
\hline 578 & $2^{2}$ & 4 & 300010095 & $580(3)$ & $\begin{array}{lcr}\mathbf{4 9 4}(96), & \mathbf{5 3 7 ( 9 6 ) ,} & 545(16), \\
561(3), & 562(12), & 563(3), \\
\mathbf{5 6 4 ( 2 4 ) ,} & 565(24), & \mathbf{5 6 7}, \\
\mathbf{5 6 8 ( 1 2 )} & & \\
\end{array}$ \\
\hline 580 & 2 & 2 & 2857239 & 581 & 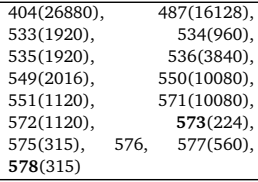 \\
\hline
\end{tabular}

Table 9: Subgroups occuring in $L_{\mathrm{IvSh}}$ extracted from $\Lambda_{\mathrm{O}^{\prime} \mathrm{N}}$ 


\begin{tabular}{|c|c|c|c|c|c|c|c|}
\hline Orbit & 1 & 2 & 3 & 4 & 5 & 6 & 7 \\
\hline Size & 1 & 1463 & 1463 & 1463 & 5852 & 12540 & 12540 \\
\hline Name & $\{p\}$ & $O_{1463}^{2}$ & $O_{1463}^{1}$ & $O_{1463}^{3}$ & & & \\
\hline
\end{tabular}

\begin{tabular}{|c|c|c|c|c|c|c|c|}
\hline Orbit & 8 & 9 & 10 & 11 & 12 & 13 & 14 \\
\hline Size & 21945 & 21945 & 29260 & 29260 & 29260 & 29260 & 29260 \\
\hline Name & $O_{21945}^{2}$ & $O_{21945}^{1}$ & & & & & \\
\hline
\end{tabular}

\begin{tabular}{|c|c|c|c|c|c|c|c|}
\hline Orbit & 15 & 16 & 17 & 18 & 19 & 20 & 21 \\
\hline Size & 58520 & 58520 & 87780 & 87780 & 87780 & 87780 & 87780 \\
\hline
\end{tabular}

\begin{tabular}{|c|c|c|c|c|c|c|c|}
\hline Orbit & 22 & 23 & 24 & 25 & 26 & 27 & 28 \\
\hline Size & 87780 & 87780 & 87780 & 87780 & 87780 & 87780 & 87780 \\
\hline
\end{tabular}

\begin{tabular}{|c|c|c|c|c|c|c|c|}
\hline Orbit & 29 & 30 & 31 & 32 & 33 & 34 & 35 \\
\hline Size & 175560 & 175560 & 175560 & 175560 & 175560 & 175560 & 175560 \\
\hline
\end{tabular}

Table 10: Orbit sizes and names of $J_{1}$ on 2624832 points (as referred to in the text)

\begin{tabular}{|c|c|c|c|c|c|c|c|c|c|c|c|c|c|c|c|c|c|}
\hline $\begin{array}{l}\text { Orbits } \\
\end{array}$ & 1 & 2 & 3 & 4 & 5 & 6 & 7 & 8 & 9 & 10 & 11 & 12 & 13 & 14 & 15 & 16 & 17 \\
\hline 1 & 0 & 1463 & 0 & 0 & 0 & 0 & 0 & 0 & 0 & 0 & 0 & 0 & 0 & 0 & 0 & 0 & 0 \\
\hline 2 & 1 & 40 & 0 & 0 & 12 & 0 & 0 & 0 & 90 & 60 & 0 & 60 & 0 & 120 & 0 & 120 & 120 \\
\hline 3 & 0 & 0 & 0 & 13 & 0 & 0 & 60 & 15 & 75 & 20 & 0 & 20 & 0 & 0 & 0 & 0 & 60 \\
\hline 4 & 0 & 0 & 13 & 0 & 0 & 0 & 60 & 15 & 15 & 20 & 0 & 20 & 0 & 0 & 0 & 0 & 0 \\
\hline 5 & 0 & 3 & 0 & 0 & 0 & 30 & 0 & 30 & 0 & 30 & 5 & 0 & 15 & 0 & 0 & 60 & 75 \\
\hline 6 & 0 & 0 & 0 & 0 & 14 & 42 & 0 & 7 & 28 & 0 & 21 & 14 & 28 & 0 & 0 & 42 & 77 \\
\hline 7 & 0 & 0 & 7 & 7 & 0 & 0 & 28 & 28 & 21 & 14 & 14 & 28 & 0 & 21 & 14 & 28 & 7 \\
\hline 8 & 0 & 0 & 1 & 1 & 8 & 4 & 16 & 26 & 19 & 28 & 16 & 20 & 20 & 32 & 8 & 56 & 64 \\
\hline 9 & 0 & 6 & 5 & 1 & 0 & 16 & 12 & 19 & 16 & 12 & 12 & 28 & 8 & 12 & 40 & 24 & 56 \\
\hline 10 & 0 & 3 & 1 & 1 & 6 & 0 & 6 & 21 & 9 & 4 & 6 & 20 & 12 & 33 & 30 & 30 & 57 \\
\hline 11 & 0 & 0 & 0 & 0 & 1 & 9 & 6 & 12 & 9 & 6 & 16 & 15 & 30 & 9 & 48 & 24 & 60 \\
\hline 12 & 0 & 3 & 1 & 1 & 0 & 6 & 12 & 15 & 21 & 20 & 15 & 22 & 12 & 48 & 18 & 36 & 66 \\
\hline 13 & 0 & 0 & 0 & 0 & 3 & 12 & 0 & 15 & 6 & 12 & 30 & 12 & 12 & 1 & 20 & 26 & 48 \\
\hline 14 & 0 & 6 & 0 & 0 & 0 & 0 & 9 & 24 & 9 & 33 & 9 & 48 & 1 & 66 & 20 & 32 & 60 \\
\hline 15 & 0 & 0 & 0 & 0 & 0 & 0 & 3 & 3 & 15 & 15 & 24 & 9 & 10 & 10 & 37 & 41 & 54 \\
\hline 16 & 0 & 3 & 0 & 0 & 6 & 9 & 6 & 21 & 9 & 15 & 12 & 18 & 13 & 16 & 41 & 40 & 45 \\
\hline 17 & 0 & 2 & 1 & 0 & 5 & 11 & 1 & 16 & 14 & 19 & 20 & 22 & 16 & 20 & 36 & 30 & 50 \\
\hline 18 & 0 & 1 & 0 & 0 & 3 & 7 & 5 & 6 & 10 & 14 & 14 & 16 & 16 & 10 & 34 & 36 & 44 \\
\hline 19 & 0 & 0 & 0 & 3 & 8 & 13 & 2 & 9 & 1 & 20 & 6 & 6 & 14 & 9 & 26 & 44 & 36 \\
\hline 20 & 0 & 1 & 2 & 2 & 0 & 4 & 6 & 16 & 14 & 13 & 14 & 14 & 19 & 14 & 34 & 34 & 42 \\
\hline 21 & 0 & 0 & 1 & 2 & 2 & 8 & 4 & 11 & 11 & 17 & 23 & 14 & 18 & 20 & 36 & 28 & 52 \\
\hline 22 & 0 & 4 & 1 & 1 & 6 & 4 & 8 & 13 & 12 & 20 & 16 & 17 & 19 & 10 & 38 & 30 & 49 \\
\hline 23 & 0 & 1 & 2 & 2 & 2 & 6 & 8 & 16 & 20 & 19 & 16 & 11 & 19 & 26 & 36 & 34 & 46 \\
\hline 24 & 0 & 0 & 1 & 2 & 2 & 4 & 16 & 13 & 9 & 10 & 20 & 17 & 18 & 7 & 30 & 30 & 33 \\
\hline 25 & 0 & 0 & 1 & 1 & 2 & 12 & 9 & 11 & 14 & 10 & 27 & 22 & 24 & 4 & 26 & 24 & 56 \\
\hline 26 & 0 & 0 & 1 & 0 & 1 & 5 & 12 & 8 & 6 & 12 & 16 & 9 & 22 & 2 & 32 & 24 & 30 \\
\hline 27 & 0 & 0 & 3 & 1 & 2 & 4 & 7 & 5 & 18 & 21 & 22 & 10 & 9 & 6 & 32 & 30 & 62 \\
\hline 28 & 0 & 1 & 0 & 0 & 5 & 9 & 7 & 6 & 16 & 16 & 16 & 17 & 16 & 22 & 36 & 36 & 56 \\
\hline 29 & 0 & 0 & 1 & 1 & 5 & 4 & 8 & 14 & 7 & 22 & 13 & 16 & 17 & 17 & 27 & 26 & 50 \\
\hline 30 & 0 & 1 & 0 & 0 & 5 & 10 & 6 & 11 & 13 & 18 & 12 & 21 & 13 & 25 & 31 & 34 & 52 \\
\hline 31 & 0 & 0 & 0 & 0 & 1 & 9 & 8 & 14 & 11 & 13 & 16 & 18 & 21 & 19 & 31 & 32 & 38 \\
\hline 32 & 0 & 1 & 1 & 1 & 4 & 9 & 3 & 10 & 13 & 17 & 13 & 11 & 17 & 15 & 36 & 32 & 53 \\
\hline 33 & 0 & 0 & 1 & 1 & 1 & 4 & 14 & 15 & 14 & 12 & 23 & 20 & 14 & 16 & 45 & 38 & 46 \\
\hline 34 & 0 & 1 & 1 & 1 & 3 & 0 & 6 & 14 & 14 & 17 & 18 & 16 & 15 & 16 & 37 & 34 & 54 \\
\hline 35 & 0 & 0 & 0 & 0 & 5 & 11 & 2 & 8 & 12 & 19 & 13 & 16 & 17 & 18 & 27 & 32 & 54 \\
\hline
\end{tabular}

Table 11: The distance distribution map of $\mathcal{G}_{\text {IvSh }}(1 / 2)$ 


\begin{tabular}{|c|c|c|c|c|c|c|c|c|c|c|c|c|c|c|c|c|c|c|}
\hline Orbits & 18 & 19 & 20 & 21 & 22 & 23 & 24 & 25 & 26 & 27 & 28 & 29 & 30 & 31 & 32 & 33 & 34 & 35 \\
\hline 1 & 0 & 0 & 0 & 0 & 0 & 0 & 0 & 0 & 0 & 0 & 0 & 0 & 0 & 0 & 0 & 0 & 0 & 0 \\
\hline 2 & 60 & 0 & 60 & 0 & 240 & 60 & 0 & 0 & 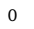 & 0 & 60 & 0 & 120 & 0 & 120 & 0 & 120 & 0 \\
\hline 3 & 0 & 0 & 120 & 60 & 60 & 120 & 60 & 60 & 60 & 180 & 0 & 120 & 0 & 0 & 120 & 120 & 120 & 0 \\
\hline 4 & 0 & 180 & 120 & 120 & 60 & 120 & 120 & 60 & 0 & 60 & 0 & 120 & 0 & 0 & 120 & 120 & 120 & 0 \\
\hline 5 & 45 & 120 & 0 & 30 & 90 & 30 & 30 & 30 & 15 & 30 & 75 & 150 & 150 & 30 & 120 & 30 & 90 & 150 \\
\hline 6 & 49 & 91 & 28 & 56 & 28 & 42 & 28 & 84 & 35 & 28 & 63 & 56 & 140 & 126 & 126 & 56 & 0 & 154 \\
\hline 7 & 35 & 14 & 42 & 28 & 56 & 56 & 112 & 63 & 84 & 49 & 49 & 112 & 84 & 112 & 42 & 196 & 84 & 28 \\
\hline 8 & 24 & 36 & 64 & 44 & 52 & 64 & 52 & 44 & 32 & 20 & 24 & 112 & 88 & 112 & 80 & 120 & 112 & 64 \\
\hline 9 & 40 & 4 & 56 & 44 & 48 & 80 & 36 & 56 & 24 & 72 & 64 & 56 & 104 & 88 & 104 & 112 & 112 & 96 \\
\hline 10 & 42 & 60 & 39 & 51 & 60 & 57 & 30 & 30 & 36 & 63 & 48 & 132 & 108 & 78 & 102 & 72 & 102 & 114 \\
\hline 11 & 42 & 18 & 42 & 69 & 48 & 48 & 60 & 81 & 48 & 66 & 48 & 78 & 72 & 96 & 78 & 138 & 108 & 78 \\
\hline 12 & 48 & 18 & 42 & 42 & 51 & 33 & 51 & 66 & 27 & 30 & 51 & 96 & 126 & 108 & 66 & 120 & 96 & 96 \\
\hline 13 & 48 & 42 & 57 & 54 & 57 & 57 & 54 & 72 & 66 & 27 & 48 & 102 & 78 & 126 & 102 & 84 & 90 & 102 \\
\hline 14 & 30 & 27 & 42 & 60 & 30 & 78 & 21 & 12 & 6 & 18 & 66 & 102 & 150 & 114 & 90 & 96 & 96 & 108 \\
\hline 15 & 51 & 39 & 51 & 54 & 57 & 54 & 45 & 39 & 48 & 48 & 54 & 81 & 93 & 93 & 108 & 135 & 111 & 81 \\
\hline 16 & 54 & 66 & 51 & 42 & 45 & 51 & 45 & 36 & 36 & 45 & 54 & 78 & 102 & 96 & 96 & 114 & 102 & 96 \\
\hline 17 & 44 & 36 & 42 & 52 & 49 & 46 & 33 & 56 & 30 & 62 & 56 & 100 & 104 & 76 & 106 & 92 & 108 & 108 \\
\hline 18 & 52 & 60 & 46 & 37 & 56 & 35 & 49 & 58 & 50 & 50 & 46 & 94 & 112 & 112 & 94 & 90 & 100 & 106 \\
\hline 19 & 60 & 86 & 51 & 60 & 41 & 39 & 56 & 46 & 52 & 49 & 48 & 104 & 98 & 78 & 118 & 80 & 88 & 112 \\
\hline 20 & 46 & 51 & 60 & 44 & 46 & 54 & 47 & 57 & 58 & 40 & 35 & 92 & 84 & 112 & 108 & 100 & 118 & 82 \\
\hline 21 & 37 & 60 & 44 & 54 & 49 & 60 & 54 & 50 & 43 & 48 & 49 & 90 & 96 & 90 & 106 & 96 & 92 & 98 \\
\hline 22 & 56 & 41 & 46 & 49 & 32 & 48 & 50 & 45 & 34 & 64 & 50 & 116 & 88 & 78 & 88 & 102 & 112 & 116 \\
\hline 23 & 35 & 39 & 54 & 60 & 48 & 58 & 48 & 33 & 56 & 48 & 44 & 92 & 88 & 104 & 104 & 102 & 92 & 94 \\
\hline 24 & 49 & 56 & 47 & 54 & 50 & 48 & 58 & 54 & 66 & 59 & 54 & 90 & 96 & 94 & 82 & 108 & 96 & 90 \\
\hline 25 & 58 & 46 & 57 & 50 & 45 & 33 & 54 & 64 & 43 & 58 & 34 & 102 & 88 & 110 & 84 & 102 & 104 & 88 \\
\hline 26 & 50 & 52 & 58 & 43 & 34 & 56 & 66 & 43 & 70 & 53 & 56 & 114 & 78 & 118 & 104 & 94 & 82 & 112 \\
\hline 27 & 50 & 49 & 40 & 48 & 64 & 48 & 59 & 58 & 53 & 52 & 50 & 94 & 100 & 86 & 108 & 92 & 98 & 82 \\
\hline 28 & 46 & 48 & 35 & 49 & 50 & 44 & 54 & 34 & 50 & 50 & 50 & 94 & 116 & 88 & 98 & 92 & 82 & 118 \\
\hline 29 & 47 & 52 & 46 & 45 & 58 & 46 & 45 & 51 & 57 & 47 & 47 & 107 & 97 & 95 & 103 & 83 & 101 & 108 \\
\hline 30 & 56 & 49 & 42 & 48 & 44 & 44 & 48 & 44 & 20 & 50 & 50 & 97 & 98 & 9 & 98 & 9 & 00 & 106 \\
\hline 31 & 56 & 39 & 56 & 45 & 39 & 52 & 47 & 55 & 39 & 43 & 44 & 95 & 98 & 111 & 92 & 107 & 93 & 101 \\
\hline 32 & 47 & 59 & 54 & 53 & 44 & 52 & 41 & 42 & 52 & 54 & 49 & 103 & 98 & 92 & 96 & 85 & 99 & 107 \\
\hline 33 & 45 & 40 & 50 & 48 & 51 & 51 & 54 & 51 & 47 & 46 & 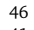 & . & 97 & 107 & 85 & 119 & 107 & 72 \\
\hline 34 & 50 & 44 & 59 & 46 & 56 & 46 & 48 & 52 & 41 & 49 & 41 & 101 & 95 & 93 & 99 & 107 & 103 & 86 \\
\hline 35 & 53 & 56 & 41 & 49 & 58 & 47 & 45 & 44 & 56 & 41 & 59 & 108 & 106 & 101 & 107 & 72 & 86 & 100 \\
\hline
\end{tabular}

Table 12: The distance distribution map of $\mathcal{G}_{\mathrm{IvSh}}(2 / 2)$ 


\section{References}

[1] M. Aschbacher, Flag structures on Tits geometries, Geom. Dedicata 14 (1983), no. 1, 21-32.

[2] C. Bates, D. Bundy, S. Hart and P. Rowley, Commuting involution graphs for sporadic simple groups, J. Algebra 316 (2007), no. 2, 849-868.

URL http://dx.doi.org/10.1016/j.jalgebra.2007.04.019

[3] W. Bosma, J. Cannon and C. Playoust, The Magma algebra system. I. The user language, J. Symbolic Comput. 24 (1997), no. 3-4, 235-265, Computational Algebra and Number Theory (London, 1993).

[4] A. E. Brouwer, A. M. Cohen and A. Neumaier, Distance Regular Graphs, Springer-Verlag, Berlin, New York, 1989.

[5] F. Buekenhout, Diagrams for geometries and groups, J. Combin. Theory Ser. A 27 (1979), no. 2, 121-151.

URL http://dx.doi .org/10.1016/0097-3165(79)90041-4

[6] _ Diagram geometries for sporadic groups, in Finite Groups - Coming of Age: proceedings of the Canadian Mathematical Society Conference held on June 15-28, 1982, vol. 45, A.M.S series Contemporary Mathematics, USA, 1985, pp. 1-32.

[7] F. Buekenhout, P. Cara and M. Dehon, Geometries of small almost simple groups based on maximal subgroups, Bulletin of the Belgian Mathematical Society, 1998.

[8] F. Buekenhout, P. Cara, M. Dehon and D. Leemans, Residually weakly primitive geometries of small sporadic and almost simple groups: a synthesis, in Topics in diagram geometry, Quad. Mat., vol. 12, Dept. Math., Seconda Univ. Napoli, Caserta, 2003, pp. 1-27.

[9] F. Buekenhout, P. Cara and K. Vanmeerbeek, Geometries of the group $\operatorname{PSL}(2,11)$, Geom. Dedicata 83 (2000), no. 1-3, 169-206, special issue dedicated to Helmut R. Salzmann on the occasion of his 70th birthday. URL http://dx.doi .org/10.1023/A:1005204612043

[10] F. Buekenhout and A. M. Cohen, Diagram geometry. Related to classical groups and buildings, Ergebnisse der Mathematik und ihrer Grenzgebiete. 3. Folge. A Series of Modern Surveys in Mathematics [Results in Mathematics and Related Areas. 3rd Series. A Series of Modern Surveys in Mathematics], vol. 57, Springer, Heidelberg, 2013.

URL http://dx . doi .org/10.1007/978-3-642-34453-4 
[11] F. Buekenhout, M. Dehon and D. Leemans, An atlas of residually weakly primitive geometries for small groups, Acad. Roy. Belg. Cl. Sci. Mém. Collect. $8^{\circ}$ (3) 14 (1999), 175 pp.

[12] F. Buekenhout and D. Leemans, On a geometry of Ivanov and Shpectorov for the O'Nan sporadic simple group, J. Combin. Theory Ser. A 85 (1999), no. $2,148-164$.

URL http://dx.doi.org/10.1006/jcta.1998.2912

[13] F. Buekenhout and A. Pasini, Finite diagram geometry extending buildings, in Handbook of Incidence Geometry: Buildings and Foundations (ed. F. Buekenhout), chapter “22”, Elsevier Science, 1995, pp. 1143-1254.

[14] P. Cara and D. Leemans, The residually weakly primitive geometries of $S_{5} \times 2$, Discrete Math. 255 (2002), no. 1-3, 35-45, Combinatorics '98 (Palermo).

URL http://dx.doi.org/10.1016/S0012-365X (01)00385-5

[15] T. Connor, A rank 3 geometry for the O'Nan group connected to the Livingstone graph, Innov. Incidence Geom. 13 (2013), 83-95.

[16] T. Connor and D. Leemans, An atlas of subgroup lattices of finite almost simple groups, Ars Math. Contemp. 8 (2015), 259-266.

[17] J. H. Conway, R. T. Curtis, S. P. Norton, R. A. Parker and R. A. Wilson, Atlas of finite groups. Maximal subgroups and ordinary characters for simple groups. With computational assistance from J. G. Thackray, Oxford University Press, Eynsham, 1985, .

[18] J. D. Dixon and B. Mortimer, Permutation groups, Graduate Texts in Mathematics, vol. 163, Springer-Verlag, New York, 1996. URL http://dx.doi .org/10.1007/978-1-4612-0731-3

[19] H. Gottschalk and D. Leemans, The residually weakly primitive geometries of the Janko group $J_{1}$, in Groups and geometries (Siena, 1996), Trends Math., Birkhäuser, Basel, 1998, pp. 65-79.

[20] J. I. Hall, Locally Petersen graphs, J. Graph Theory 4 (1980), no. 2, 173187.

URL http://dx.doi.org/10.1002/jgt.3190040206

[21] F. Harary, Graph theory, Addison-Wesley Publishing Co., Reading, Mass.Menlo Park, Calif.-London, 1969. 
[22] M. I. Hartley, I. Hubard and D. Leemans, Regular 4-polytopes from the Livingstone graph of Janko's first group, J. Algebraic Combin. 35 (2012), no. 2, 193-214.

URL http://dx.doi.org/10.1007/s10801-011-0300-x

[23] W. Imrich, S. Klavžar and A. Vesel, A characterization of halved cubes, Ars Combin. 48 (1998), 27-32.

[24] A. A. Ivanov and S. V. Shpektorov, A geometry for the O'Nan-Sims group, connected with the Petersen graph, Uspekhi Mat. Nauk 41 (1986), no. 3(249), 183-184.

[25] A. A. Ivanov, S. V. Tsaranov and S. V. Shpektorov, Maximal subgroups of the O'Nan-Sims sporadic simple group and its automorphism group, Dokl. Akad. Nauk SSSR 291 (1986), no. 4, 777-780.

[26] D. Leemans, On computing the subgroup lattice of $O^{\prime} N$, Unpublished manuscript (2008), 1-23.

http://www.math. auckland.ac.nz/ dleemans/abstracts/onlat.html

[27]___ Residually weakly primitive and locally two-transitive geometries for sporadic groups, Académie Royale de Belgique: Classe des Sciences, vol. XI, 2058, 2008.

[28] D. Livingstone, On a permutation representation of the Janko group, $J$. Algebra 6 (1967), 43-55.

[29] M. E. O'Nan, Some evidence for the existence of a new simple group, Proc. London Math. Soc. (3) 32 (1976), no. 3, 421-479.

[30] M. Perkel, A characterization of $J_{1}$ in terms of its geometry, Geom. Dedicata 9 (1980), no. 3, 291-298.

[31] S. V. Shpectorov, On Geometries with diagram $P^{n}$, Ph.D. thesis, University of Moscow (1989).

[32] L. H. Soicher, Presentations of some finite groups with applications to the O'Nan simple group, J. Algebra 108 (1987), no. 2, 310-316. URL http://dx.doi .org/10.1016/0021-8693(87)90104-9

[33] J. Tits, Sur la géométrie des R-espaces, J. Math. Pures Appl. (9) 36 (1957), 17-38.

[34] _ Sur les analogues algébriques des groupes semi-simples complexes, in Colloque d'algèbre supérieure, tenu à Bruxelles du 19 au 22 décembre 1956, Centre Belge de Recherches Mathématiques, 
Établissements Ceuterick, Louvain; Librairie Gauthier-Villars, Paris, 1957, pp. 261-289.

[35] E. W. Weisstein, Locally Petersen Graph, From MathWorld-A Wolfram Web Resource.

[36] R. A. Wilson, The maximal subgroups of the O'Nan group, J. Algebra 97 (1985), no. 2, 467-473.

[37] S. Yoshiara, The maximal subgroups of the sporadic simple group of O’Nan, J. Fac. Sci. Univ. Tokyo Sect. IA Math. 32 (1985), no. 1, 105-141.

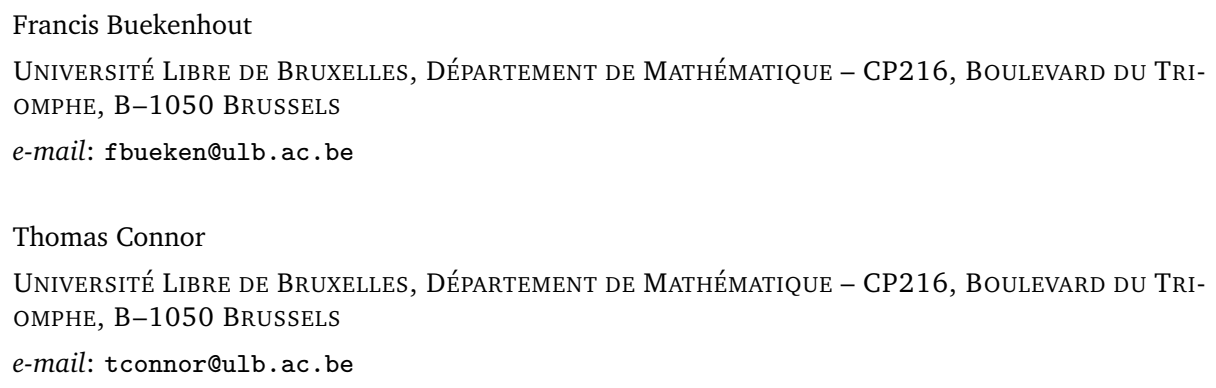

